\title{
Jülich hyperon-nucleon model revisited
}

\author{
J. Haidenbauer ${ }^{1}$ and Ulf-G. Meißner ${ }^{1,2}$ \\ ${ }^{1}$ Institut für Kernphysik (Theorie), Forschungszentrum Jülich, D-52425 Jülich, Germany, \\ ${ }^{2}$ Helmholtz-Institut für Strahlen- und Kernphysik (Theorie), Universität Bonn, Nußallee 14-16, D-53115 Bonn, Germany
}

(Received 9 June 2005; published 28 October 2005)

\begin{abstract}
A one-boson-exchange model for the hyperon-nucleon $(\Lambda N, \Sigma N)$ interaction is presented. The model incorporates the standard one boson exchanges of the lowest pseudoscalar and vector meson multiplets with coupling constants fixed by SU(6) flavor symmetry relations. As the main new feature of the model, the contributions in the scalar-isoscalar $(\sigma)$ and vector-isovector $(\rho)$ exchange channels are now constrained by a microscopic model of correlated $\pi \pi$ and $K \bar{K}$ exchange. Additional short-ranged ingredients of the model in the scalar-isovector $\left(a_{0}\right)$ and scalar-isospin-1/2 $(\kappa)$ channels are likewise viewed as arising from meson-meson correlations but are treated phenomenologically. With this model a satisfactory reproduction of the available hyperon-nucleon data can be achieved.
\end{abstract}

DOI: 10.1103/PhysRevC.72.044005 PACS number(s): 13.75.Ev, 12.39.Pn, 21.30.-x, 21.80.+a

\section{INTRODUCTION}

The role of strangeness in low and medium energy nuclear physics is currently of considerable interest, as it has the potential to deepen our understanding of the relevant strong interaction mechanisms in the nonperturbative regime of QCD. For example, the system of a strange baryon (hyperon $Y$ ) and a nucleon $(N)$ is in principle an ideal testing ground for investigating the importance of $\mathrm{SU}(3)_{\text {flavor }}$ symmetry in hadronic interactions. Existing meson exchange models of the $Y N$ force usually assume $\mathrm{SU}(3)$ symmetry for the hadronic coupling constants, and in some cases [1,2] even the SU(6) symmetry of the quark model. The symmetry requirements provide relations between couplings of mesons of a given multiplet to the baryon current, which greatly reduce the number of free model parameters. Specifically, coupling constants at the strange vertices are then connected to nucleon-nucleon-meson coupling constants, which in turn are constrained by the wealth of empirical information on $N N$ scattering. Essentially all these $Y N$ interaction models can reproduce the existing $Y N$ scattering data, so that at present the assumption of SU(3) symmetry for the coupling constants cannot be ruled out by experiment.

One should note, however, that the various models differ dramatically in the treatment of the scalar-isoscalar meson sector, which describes the baryon-baryon interaction at intermediate ranges. For example, the Nijmegen group [3-6] views this interaction as being generated by genuine scalar meson exchange. In their model D [3] an $\epsilon(760)$ is exchanged as an $\mathrm{SU}(3)_{\text {flavor }}$ singlet. In models F [4], NSC [5], and NSC97 [6] a scalar SU(3) nonet is exchanged-namely, two isospin- 0 mesons [besides the $\epsilon(760)$, the $\epsilon^{\prime}(1250)$ in model $\mathrm{F}$ and $S^{*}(975)\left(f_{0}(980)\right)$ in model NSC (NSC97)], an isospin- 1 meson [ $\delta$ or $\left.a_{0}(980)\right]$ and an isospin-1/2 strange meson $\kappa$ with a mass of $1000 \mathrm{MeV}$. A genuine scalar SU(3) nonet is also present in the so-called Ehime potential [7], where in addition to the $S^{*}(975)$ and $\delta$ [or $a_{0}(980)$ ] the $f_{0}(1581)$ and the $K_{0}^{*}(1429)$ are included. In addition the model incorporates two effective scalar-meson exchanges, $\sigma(484)$ and $\kappa(839)$, that stand for $(\pi \pi)_{I=0}$ and $(K \pi)_{I=1 / 2}$ correlations but are treated phenomenologically. The Tübingen model, on the other hand, which is essentially a constituent quark model supplemented by $\pi$ and $\sigma$ exchange at intermediate and short ranges, treats the $\sigma$ meson as an $\mathrm{SU}(3)$ singlet with a mass of $520 \mathrm{MeV}$ [8] or $675 \mathrm{MeV}$ [9], respectively. Finally, in the quark-models of Zhang et al. [10] and Fujiwara et al. [11] a scalar SU(3) nonet is exchanged, though in this case between quarks and not between the baryons.

In the (full) Bonn $N N$ potential [12] the intermediate range attraction is provided by uncorrelated and correlated $\pi \pi$ exchange processes [Figs. 1(a)-1(b) and 1(c), respectively], with $N N, N \Delta$ and $\Delta \Delta$ intermediate states. From earlier studies of the $\pi \pi$ interaction it is known that $\pi \pi$ correlations are important mainly in the scalar-isoscalar and vectorisovector channels. In one-boson-exchange (OBE) potentials these are included effectively via exchange of sharp mass $\sigma$ and $\rho$ mesons. One disadvantage of such a simplified treatment is that this parametrization cannot be transferred into the hyperon sector in a well-defined manner. Therefore in the earlier $Y N$ interaction models of the Jülich group [1], which start from the Bonn $N N$ potential, the coupling constants of the fictitious $\sigma$ meson at the strange vertices $(\Lambda \Lambda \sigma, \Sigma \Sigma \sigma)$ are free parameters-a rather unsatisfactory feature of the models. This is especially true for the extension to the strangeness $S=-2$ channels, interest in which initiated with the prediction of the H-dibaryon by Jaffe [13].

These problems can be overcome by an explicit evaluation of correlated $\pi \pi$ exchange in the various baryon-baryon channels. A corresponding calculation was initially done only for the $N N$ case [Fig. 1(c)] in Ref. [14], but was extended in a recent paper [15] by the Jülich group so that now a full and consistent microscopic derivation of correlated $\pi \pi$ exchange in various baryon-baryon $\left(B B^{\prime}\right)$ channels with strangeness $S=0,-1$, and -2 is available. The starting point was a field theoretical model for both the $N \bar{N} \rightarrow \pi \pi$ Born amplitudes and the $\pi \pi$ and $K \bar{K}$ elastic scattering [16-18]. Thus, the $K \bar{K}$ channel is treated on an equal footing with the $\pi \pi$ channel in order to reliably determine the influence of $K \bar{K}$ correlations in the relevant $t$-channels. Then, with the help of unitarity and dispersion relations the amplitude for the correlated $\pi \pi$ 




(a)

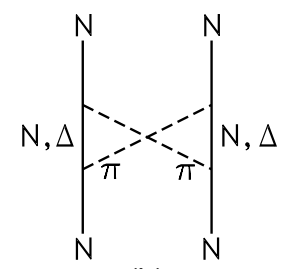

(b)

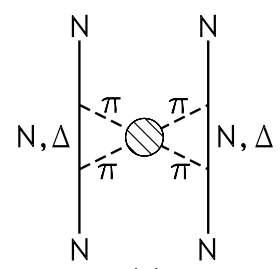

(c)
FIG. 1. Two-pion exchange in the $N N$ interaction: (a) uncorrelated iterative and (b) crossed boxes, (c) correlated two-pion exchange.

exchange in the $N N$ channel but also for the $Y N$ and $Y Y$ systems were computed. Thus, within this approach one can replace the phenomenological $\sigma$ and $\rho$ exchanges in the Bonn $N N$ [12] and Jülich $Y N$ [1] models by correlated processes, i.e., eliminate undetermined parameters such as the $B B^{\prime} \sigma$ coupling constants.

In the present paper a new $Y N$ model is presented that utilizes this microscopic model of correlated $\pi \pi$ and $K \bar{K}$ exchange to fix the contributions in the scalar-isoscalar $(\sigma)$ and vector-isovector $(\rho)$ channels. The model incorporates also the standard one boson exchange contributions of the lowest pseudoscalar and vector meson multiplets with coupling constants determined by $\mathrm{SU}(6)$ symmetry relations. Assuming the SU(6) symmetry means that also the so-called $F /(F+D)$ ratios are fixed. In addition, there are further new ingrediations as compared to the original Jülich $Y N$ model [1]. First of all, the contribution from the $a_{0}(980)$ meson is taken into account. Secondly, we consider the exchange of a strange scalar meson, the $\kappa$, with mass $\sim 1000 \mathrm{MeV}$. Let us emphasize, however, that in analogy with the $\sigma$ meson these particles are likewise not viewed as being members of a scalar meson SU(3) multiplet, but rather as representations of strong meson-meson correlations in the scalar-isospin-1/2 $(\pi K)$ [16] and scalarisovector $(\pi \eta-K \bar{K})$ [17] channels, respectively. In principle, their contributions can also be evaluated along the lines of Ref. [15], however, for simplicity in the present model they are effectively parametrized by one-boson-exchange diagrams with the appropriate quantum numbers assuming the coupling constants to be free parameters.

In the next two sections we describe the principal steps of the derivation of correlated $\pi \pi$ and $K \bar{K}$ exchange potentials for the baryon-baryon amplitudes in the $\sigma$ and $\rho$ channels. In particular, in Sec. II we give a short outline of the microscopic model for the required $B \overline{B^{\prime}} \rightarrow \pi \pi, K \bar{K}$ amplitudes. The derivation of the potentials themselves is indicated in Sec. III. Furthermore, we introduce and discuss the parametrization of correlated $\pi \pi$ and $K \bar{K}$ exchange potentials by an effective $\sigma$ and $\rho$ exchange for the $Y N$ channels. These effective parametrizations are then adopted for the construction of the new $Y N$ model. In Sec. IV the other ingredients of our $Y N$ model are introduced. Specifically, we comment on the employed strategy for fixing the parameters of the model. Then we present and discuss numerical results of the model for $Y N$ scattering observables, phase shifts and effective range parameters. Finally, some concluding remarks are made in Sec. V.

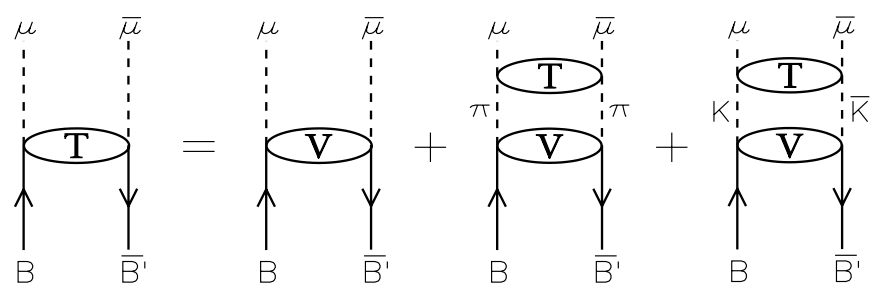

FIG. 2. The dynamical model for the $B \bar{B} \rightarrow \mu \bar{\mu}$ amplitude $(\mu \bar{\mu}=\pi \pi, K \bar{K})$.

\section{MODEL FOR CORRELATED $2 \pi$ EXCHANGE}

Based on a $\pi \pi-K \bar{K}$ amplitude the evaluation of diagrams such as in Fig. 1(c) for any $B B^{\prime}$ system can be done in two steps. Firstly the $N \bar{N}(\Lambda \bar{\Lambda}, \Sigma \bar{\Sigma}$, etc. $) \rightarrow 2 \pi, K \bar{K}$ amplitudes are determined in the pseudophysical region $\left(t \leqslant 4 m_{\pi}^{2}\right)$ and then dispersion theory and unitarity are applied to connect those amplitudes with the corresponding physical amplitudes in the various baryon-baryon channels.

Figure 2 shows a graphic representation of our dynamical model for correlated $2 \pi-K \bar{K}$ exchange. Here $B \overline{B^{\prime}}$ stands for $N \bar{N}, \Lambda \bar{\Lambda}, \Lambda \bar{\Sigma} / \Sigma \bar{\Lambda}$, or $\Sigma \bar{\Sigma}$. Formally the amplitudes for the processes $B \overline{B^{\prime}} \rightarrow \alpha$ (with $\alpha=\pi \pi, K \bar{K}$ ) are obtained from solving the scattering equation

$$
T_{B, \overline{B^{\prime}} \rightarrow \alpha}=V_{B, \overline{B^{\prime}} \rightarrow \alpha}+\sum_{\beta=\pi \pi, K \bar{K}} T_{\alpha, \beta} G_{\beta} V_{B, \overline{B^{\prime}} \rightarrow \beta} .
$$

Here $T_{\alpha, \beta}$ is the $\pi \pi-K \bar{K}$ (coupled-channel) reaction amplitude, $V_{B, \overline{B^{\prime}} \rightarrow \beta}$ the $B \overline{B^{\prime}} \rightarrow \pi \pi, K \bar{K}$ transition Born amplitude and $G_{\beta}$ the free $(\pi \pi$ or $K \bar{K})$ Green's function The first two quantities are the basic ingredients of the model. These amplitudes have to be known in the so-called pseudophysical region, i.e., for energies below the $B \overline{B^{\prime}}$ threshold. While for $N \bar{N} \rightarrow \pi \pi$ the corresponding amplitudes can be derived from empirical information on $\pi N$ and $\pi \pi$ scattering via an analytic continuation, this is not possible for the transitions $Y \overline{Y^{\prime}} \rightarrow$ $\pi \pi, K \bar{K}$. Thus, a microscopic model for the $B \overline{B^{\prime}} \rightarrow \pi \pi, K \bar{K}$ is a prerequisite for the evaluation of the correlated $\pi \pi$ and $K \bar{K}$ exchange in the $Y N$ and $Y Y$ channels. Such a model was constructed by Reuber et al. in Ref. [15]. A main feature of this model is the completely consistent treatment of its two components, namely the $B \overline{B^{\prime}} \rightarrow \pi \pi, K \bar{K}$ Born amplitudes and the $\pi \pi-K \bar{K}$ correlations with respect to the $\sigma$ - and $\rho$-channels. Both components are derived in field theory from an ansatz for the hadronic Lagrangians [15]. The considered contributions are briefly described in the subsections below. The subsequent evaluation of the baryon-baryon interaction via dispersion theory and unitarity is summarized in the next section.

\section{A. The $\pi \pi-K \bar{K}$ amplitudes}

The dynamical model used for the $\pi \pi-K \bar{K}$ amplitudes is derived within the meson exchange framework and involves the $\pi \pi$ and $K \bar{K}$ coupled channels [16-18]. The driving terms for the diagonal interactions consist of ( $t$-channel) exchange diagrams ( $\rho$ and $\rho, \omega, \phi$, respectively) and ( $s$-channel) pole diagrams with $\epsilon \equiv f_{0}(1440), \rho \equiv \rho(770)$, and $f_{2} \equiv f_{2}(1274)$ 

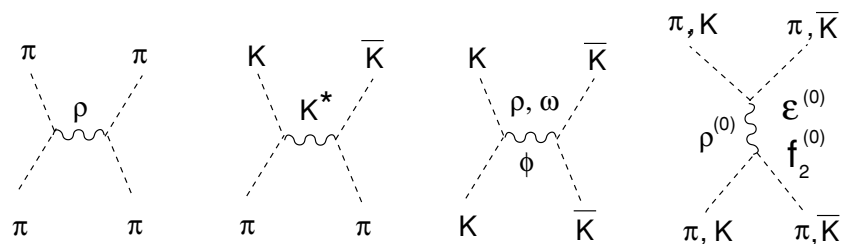

FIG. 3. The contributions to the potential of the coupled channel $\pi \pi-K \bar{K}$ model of Ref. [18].

intermediate states. The coupling $\pi \pi \rightarrow K \bar{K}$ is provided by $K^{*}(892)$ exchange. The corresponding diagrams are shown in Fig. 3. The potentials derived from those diagrams are iterated in a coupled-channel Lippmann-Schwinger-type scattering equation. The free parameters of the $\pi \pi-K \bar{K}$ model were adjusted to the empirical $\pi \pi$ phase shifts and inelasticities. For details on the model and a comparision of the resulting $\pi \pi$ phase shifts with experimental values we refer the reader to Refs. [17,18].

\section{B. The $B \bar{B} \rightarrow 2 \pi, K \bar{K}$ Born amplitudes}

The Born amplitudes for the transition $B \bar{B} \rightarrow \alpha$ with $\alpha=\pi \pi, K \bar{K}$ are built up from an (s-channel) $\rho$-pole diagram and all possible diagrams involving the exchange of baryons out of the $J^{P}=\frac{1}{2}^{+}$octet or the $J^{P}=\frac{3}{2}^{+}$decuplet [15]. For illustration we show in Fig. 4 those diagrams that contribute to the transition amplitude for $\Sigma \bar{\Sigma} \rightarrow 2 \pi, K \bar{K}$.

In the construction of the model the number of free parameters has been kept to a minimum. Specifically, the coupling constants at the various vertices involving the pseudoscalar mesons were fixed by SU(6) symmetry relations. As far as the $\rho$-pole diagram is concerned the (bare) coupling constants and form factors at the $\pi \pi \rho^{(0)}$ and $K \bar{K} \rho^{(0)}$ vertices were already determined in the model for the $\pi \pi-K \bar{K}$ interaction [18] and were taken over from there. Then, assuming that the bare $\rho$-meson couples universally to the isospin current all vector couplings $g_{B B^{\prime} \rho}^{(0)}$ to the baryonic vertices were fixed as well. For the tensor couplings $f_{B B^{\prime} \rho}^{(0)}$ again $\mathrm{SU}(6)$ symmetry relations were applied.
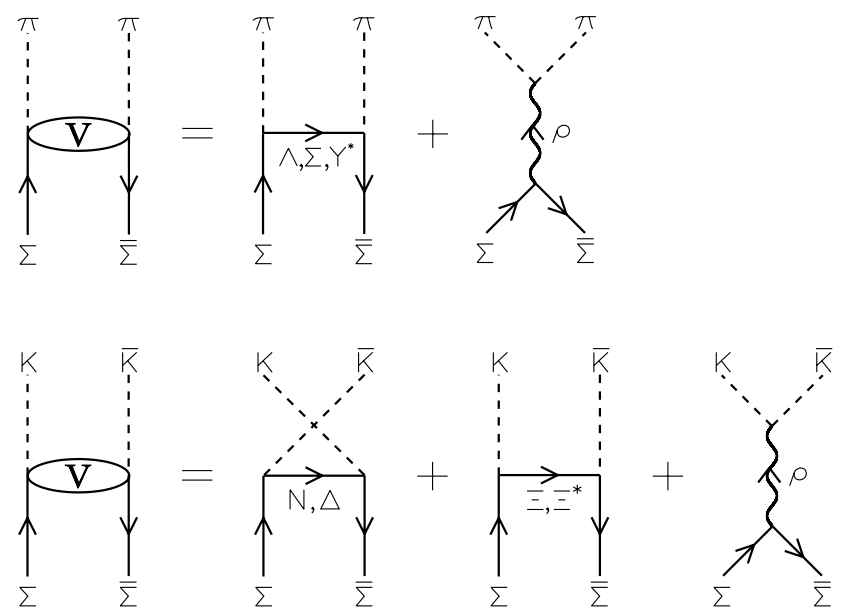

FIG. 4. The contributions to the Born amplitude for the transitions $\Sigma \bar{\Sigma} \rightarrow \pi \pi, K \bar{K}$.
The four remaining free parameters (the tensor coupling constant $f_{N N \rho}^{(0)}$, the parameter $x_{\Delta}$ characterizing the strength of the off-shell part in the $\Delta N \pi$ Lagrangian [59], and formfactor parameters for the exchanged baryons for the octet and decuplet, respectively [15]) were fixed by adjusting the model predictions to the quasi-empirical information on the amplitudes $N \bar{N} \rightarrow \pi \pi$ obtained by Höhler et al. [19] by analytically continuing the $\pi N$ and $\pi \pi$ scattering data. Once this is done the model can be used to generate the amplitudes for any $B \bar{B}^{\prime} \rightarrow \pi \pi, K \bar{K}$ channel. Though it should have become clear already from the discussion above, we want to emphasize again that the extrapolation of the model for the $N \bar{N} \rightarrow \pi \pi$ amplitudes to other channels depends crucially on the assumption of SU(3) symmetry for the pseudoscalar sector and it is based also on the hope that the correct description of the quasiempirical $N \bar{N} \rightarrow \pi \pi$ amplitudes guarantees a reasonable description of the other baryon-antibaryon channels, for which no empirical information is available.

\section{POTENTIAL FROM CORRELATED $\pi \pi$ AND $K \bar{K}$ EXCHANGE}

Assuming analyticity for the amplitudes dispersion relations can be formulated for the baryon-baryon amplitudes, which connect physical amplitudes in the $s$-channel with singularities and discontinuities of these amplitudes in the pseudophysical region of the $t$-channel processes for the $J^{P}=0^{+}(\sigma)$ and $1^{-}(\rho)$ channel:

$$
V_{B_{1}, B_{2} \rightarrow B_{1}^{\prime}, B_{2}^{\prime}}^{\left(0^{+}, 1^{-}\right)}(t) \propto \int_{4 m_{\pi}^{2}}^{\infty} d t^{\prime} \frac{\operatorname{Im} V_{B_{1}, \overline{B_{1}^{\prime}} \rightarrow \overline{B_{2}, B_{2}^{\prime}}}^{\left(0^{+}, 1^{-}\right)}}{t^{\prime}-t}, t<0 .
$$

Via unitarity relations the singularity structure of the baryonbaryon amplitudes for $\pi \pi$ and $K \bar{K}$ exchange are fixed by and can be written as products of the $B \overline{B^{\prime}} \rightarrow \pi \pi, K \bar{K}$ amplitudes

$$
\operatorname{Im} V_{B_{1}, \overline{B_{1}^{\prime}} \rightarrow \overline{B_{2}}, B_{2}^{\prime}}^{\left(0^{+} 1^{-}\right)}\left(t^{\prime}\right) \propto \sum_{\alpha=\pi \pi, K \bar{K}} T_{B_{1}, \overline{B_{1}^{\prime}} \rightarrow \alpha}^{*,\left(0^{+}, 1^{-}\right)} T_{\overline{B_{2}}, B_{2}^{\prime} \rightarrow \alpha}^{\left(0^{+}, 1^{-}\right)} .
$$

Thus, from the $B \overline{B^{\prime}} \rightarrow 2 \pi$ helicity amplitudes the spectral functions can be calculated

$$
\rho_{B_{1}, B_{2} \rightarrow B_{1}^{\prime}, B_{2}^{\prime}}^{\left(0^{+}, 1^{-}\right)}\left(t^{\prime}\right) \sum_{\alpha=\pi \pi, K \bar{K}} T_{B_{1}, \bar{B}_{1}^{\prime} \rightarrow \alpha}^{*,\left(0^{+}, 1^{-}\right)} T_{\bar{B}_{2}, B_{2}^{\prime} \rightarrow \alpha}^{\left(0^{+}, 1^{-}\right)}
$$

which are then inserted into dispersion integrals to obtain the (on-shell) baryon-baryon interaction:

$$
V_{B_{1}, B_{2} \rightarrow B_{1}^{\prime}, B_{2}^{\prime}}^{\left(0^{+}, 1^{-}\right)}(t) \propto \int_{4 m_{\pi}^{2}}^{\infty} d t^{\prime} \frac{\rho_{B_{1}, B_{2} \rightarrow B_{1}^{\prime}, B_{2}^{\prime}}^{\left(0^{+}, 1^{-}\right)}}{t^{\prime}-t}, t<0 .
$$

The underlying formalism is quite involved and has been outlined in detail already in Ref. [15]. Thus, we refrain from repeating it here. Rather we want to provide only some more general information.

Since the dispersion-theoretical evaluation is restricted to the contribution of (correlated) $\pi \pi$ and $K \bar{K}$ exchange to the baryon-baryon amplitudes only those singularities are taken into account which are generated by $\pi \pi$ and $K \bar{K}$ intermediate states, namely the discontinuities due to the $\pi \pi$ and $K \bar{K}$ unitarity cut (the so-called right-hand cut). The left-hand 
cuts, which are due to unitarity constraints for the $u$-channel reaction, can be neglected in the baryon-baryon channels considered here, since they start at large, negative $t$-values (from which they extend to $-\infty$ ) and are therefore far away from the physical region relevant for low-energy $s$-channel processes.

The $B \overline{B^{\prime}} \rightarrow \alpha$ amplitudes, which enter in Eq. (3) are derived from a microscopic model which is based on the hadron-exchange picture, cf. Sec. II. Of course, this model has a limited range of validity: for energies far beyond $t_{\max }^{\prime} \approx$ $100 m_{\pi}^{2}$ it cannot provide reliable results. The dispersion integral for the invariant amplitudes extending in principle along the whole $\pi \pi$ right-hand cut has therefore to be limited to an upper bound, $t_{\max }^{\prime}$, which has been put to $t_{\max }^{\prime}=120 \mathrm{~m}_{\pi}^{2}$ in Ref. [15].

The spectral function (4) for the $\left(0^{+}\right) \sigma$-channel has only one component but the one for the $\left(1^{-}\right) \rho$-channel consists of four linearly independent components, which reflects the more complicated spin structure of this channel.

Finally, we should note that the helicity amplitudes obtained according to Fig. 2 still generate the uncorrelated [first diagram on the right-hand side (r.h.s.) of Fig. 2], as well as the correlated pieces (second and third diagrams). Thus, in order to obtain the contribution of the truely correlated $\pi \pi$ and $K \bar{K}$ exchange one must eliminate the former from the spectral function. This is done by calculating the spectral function generated by the Born term and subtracting it from the total spectral function:

$$
\rho^{\left(0^{+}, 1^{-}\right)} \longrightarrow \rho^{\left(0^{+}, 1^{-}\right)}-\rho_{\text {Born }}^{\left(0^{+}, 1^{-}\right)}
$$

In practice this means that, e.g., for the full Bonn $N N$ model contributions involving spin-1/2 as well as spin-3/2 baryons have to be subtracted since corresponding contributions are already treated explicitly in the s-channel in this model, namely via box diagrams with intermediate $\Delta$-states as shown in Fig. 1(a). On the other hand only uncorrelated contributions involving spin-1/2 baryons are to be subtracted from the discontinuities of the invariant baryon-baryon amplitudes in order to avoid double counting if a simple OBE-model is used in the $s$-channel. This is the relevant procedure for the $Y N$ model that will be presented in the next section.

Note that the spectral functions characterize both the strength and range of the interaction. Clearly, for sharp mass exchanges the spectral function becomes a $\delta$-function at the appropriate mass.

For convenience the authors of Ref. [15] have presented their results in terms of effective coupling strengths, by parametrizing the correlated processes by (sharp mass) $\sigma$ and $\rho$ exchanges. The interaction potential resulting from the exchange of a $\sigma$ meson with mass $m_{\sigma}$ between two $J^{P}=1 / 2^{+}$ baryons $A$ and $B$ has the structure:

$$
V_{A, B \rightarrow A, B}^{\sigma}(t)=g_{A A \sigma} g_{B B \sigma} \frac{F_{\sigma}^{2}(t)}{t-m_{\sigma}^{2}},
$$

where a form factor $F_{\sigma}(t)$ is applied at each vertex, taking into account the fact that the exchanged $\sigma$ meson is not on its mass shell. This form factor is parametrized in the conventional monopole form,

$$
F_{\sigma}(t)=\frac{\Lambda_{\sigma}^{2}-m_{\sigma}^{2}}{\Lambda_{\sigma}^{2}-t},
$$

with a cutoff mass $\Lambda_{\sigma}$ assumed to be the same for both vertices. The correlated potential as given in Eq. (2) can now be parametrized in terms of $t$-dependent strength functions $G_{B_{1}^{\prime}, B_{2}^{\prime} \rightarrow B_{1}, B_{2}}(t)$, so that for the $\sigma$ case:

$$
V_{A, B \rightarrow A, B}^{\left(0^{+}\right)}(t)=G_{A B \rightarrow A B}^{\sigma}(t) F_{\sigma}^{2}(t) \frac{1}{t-m_{\sigma}^{2}} .
$$

The effective coupling constants are then defined as

$g_{A A \sigma} g_{B B \sigma} \longrightarrow G_{A B \rightarrow A B}^{\sigma}(t)=\frac{\left(t-m_{\sigma}^{2}\right)}{\pi F_{\sigma}^{2}(t)} \int_{4 m_{\pi}^{2}}^{\infty} \frac{\rho_{A B \rightarrow A B}^{\left(0^{+}\right)}\left(t^{\prime}\right)}{t^{\prime}-t} d t^{\prime}$.

Similar relations can be also derived for the correlated exchange in the isovector-vector channel [15], which in this case will involve vector as well as tensor coupling pieces.

It should be stressed that, so far, this parametrization does not involve any approximations as long as the full $t$-dependence of the effective coupling strengths is taken into account. The parameters of the $\sigma$ and $\rho$ exchange have been chosen to have the same values in all particle channels. The masses $m_{\sigma}$ and $m_{\rho}$ of the exchanged particles have been set to the values used in the Bonn-Jülich models of the $N N$ [12] and $Y N$ [1] interactions, $m_{\sigma}=550 \mathrm{MeV}, m_{\rho}=770 \mathrm{MeV}$. The cutoff masses $\Lambda_{\sigma}$ and $\Lambda_{\rho}$ have been chosen so that the coupling strengths in the $S=0,-1$ baryon-baryon channels vary only weakly with $t$. The resulting values $\left(\Lambda_{\sigma}=2.8 \mathrm{GeV}, \Lambda_{\rho}=2.5 \mathrm{GeV}\right)$ are quite large compared to the values of the phenomenological parametrizations used in Refs. [1,12], and thus represent rather hard form factors.

Note that in the OBE framework the contribution of a genuine $[\mathrm{SU}(3)] \sigma$ meson to the three reactions $N N \rightarrow$ $N N, Y N \rightarrow Y N, Y Y \rightarrow Y Y$ is determined by two parameters (coupling constants), namely $g_{N N \sigma}$ and $g_{Y Y \sigma}$, whereas the correlated exchange is characterized by three independent strength functions $\left(G_{N N \rightarrow N N}, G_{Y N \rightarrow Y N}, G_{Y Y \rightarrow Y Y}\right)$ so that vertex coupling constants cannot be determined uniquely. This implies directly that the strength parameters cannot fulfill $\mathrm{SU}(3)$ relations.

In the physical region the strength of the contributions is to a large extent governed by the value of $G$ at $t=0$. Those values for the various channels were tabulated in Ref. [15] (cf. Tables V-VII) for the case of the full model calculation and also when uncorrelated contributions involving spin-1/2 baryons only are subtracted from the spectral function of the invariant baryon-baryon amplitudes. The latter are the proper values to be used for constructing a $Y N$ model based on simple OBE-exchange diagrams.

In principle, the average size of the effective coupling strengths is only an approximate measure of the strength of correlated $\pi \pi$ and $K \bar{K}$ exchange in the various particle channels. The precise energy dependence of the correlated exchange as well as its relative strength in the different partial waves of the $s$-channel reaction is determined by the spectrum of exchanged invariant masses, or spectral functions, 
leading to a different $t$-dependence of the effective coupling strengths. This was demonstrated in Ref. [20] where the on-shell $N N, \Lambda N$, and $\Sigma N$ potentials in spin-singlet states with angular momentum $L=0,2$, and 4, generated directly by the scalar-isoscalar part of the correlated $\pi \pi$ and $K \bar{K}$ exchange, were compared to the corresponding results based on a $\sigma$ exchange with sharp mass. It could be seen that the correlated $2 \pi$ exchange is significantly stronger in high partial waves because the $\sigma$ exchange, which corresponds to a spectral function proportional to $\delta\left(t^{\prime}-m_{\sigma}^{2}\right)$, does not contain the long-range part of the correlated processes. Thus, parametrizing the results derived from the microscopic model by $\sigma$ exchange with a sharp mass, but using the effective coupling strength $G_{N N \rightarrow N N}^{\sigma}$ at $t=0$ one can obtain rough agreement with the exact result in the $S$ waves, say, but usually underestimates the magnitude considerably in the high partial waves. Obviously the replacement of correlated $\pi \pi$ and $K \bar{K}$ exchanges by an exchange of a sharp mass $\sigma$ meson with a $t$-independent coupling cannot provide a simultaneous description of both low and high partial waves.

These features are important for investigations of the $N N$ systems where the phase shifts are known quantitatively even for rather high partial waves. In this case the results of the correlated exchange should be used directly [14]. However, for the $\Lambda N$ and $\Sigma N$ systems only scattering observables are available, and those (total and differential cross sections) are primarily sensitive to $S$ - and $P$-wave contributions. Thus, here it is reasonable to simplify the calculation and use only an effective parametrization of the results derived from the microscopic model in terms of a $\sigma$ and $\rho$ exchange with a sharp mass. Specifically, combining Eqs. (7) and (9) we use the expression

$$
V_{A, B \rightarrow A, B}^{\left(0^{+}\right)}(t)=G_{A B \rightarrow A B}^{\sigma} \tilde{F}_{\sigma}^{2}(t) \frac{1}{t-m_{\sigma}^{2}}
$$

with

$$
\tilde{F}_{\sigma}(t)=\frac{\Lambda_{\sigma}^{2}}{\Lambda_{\sigma}^{2}-t}
$$

and a similar one for the $\rho$ exchange contribution. The effective coupling strength $G_{Y N \rightarrow Y^{\prime} N}^{\sigma}$ (and ${ }^{i j} G_{Y N \rightarrow Y^{\prime} N}^{\rho}$ ) is deduced via Eq. (10) (and via a similar one for the $\rho$ channel, cf. Ref. [15]) for the form factor (12) and adjusted to the value at $t=0$. The different prescription for the vertex form factor as compared to Ref. [15], i.e., to Eq. (8), is adopted here because it guarantees that the on-shell behavior of the potential (which is fully determined by the dispersion integral) is not modified strongly as long as the energy is not too high. At the same time smaller cutoff masses as those mentioned above (and employed in Ref. [15]) can be used to ensure sufficient convergence when the potential (11) is iterated in the scattering equation. The concrete values used for the cutoff masses are $\Lambda_{\sigma}=2.5$ (1.6) $\mathrm{GeV}$ for the $\Lambda N(\Sigma N)$ channels and $\Lambda_{\rho}=1.25(1.8) \mathrm{GeV}$ for the $\Lambda N \rightarrow \Sigma N$ transition ( $\Sigma N$ channel).

The effective coupling strengths employed in our new $Y N$ model are compiled in Table I. Though these values differ slightly from those given in Tables 5-7 of Ref. [15], due to the different choice of the form factor, we would like to emphasize that the strengths of the interactions at $t=0$ are
TABLE I. Effective $\sigma$ and $\rho$ coupling strengths $G_{Y N \rightarrow Y^{\prime} N}(t=0)$ for correlated $\pi \pi$ and $K \bar{K}$ exchange in the various nucleon-hyperon channels. $V V, V T$, etc., stand for the vector-vector, vector-tensor, etc., combinations of the $\rho$ coupling, cf. Ref. [15].

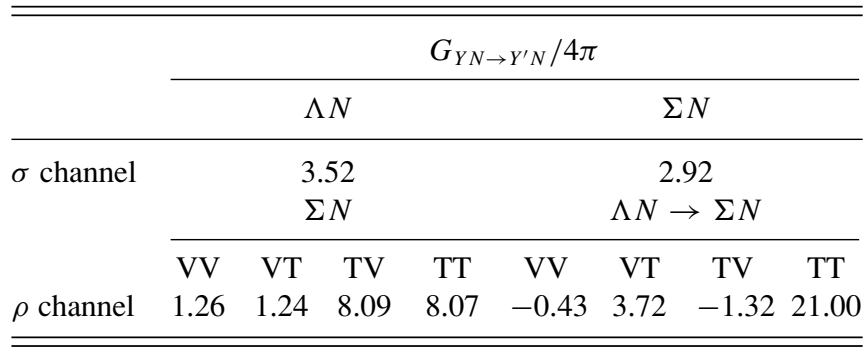

the same in both cases and coincide with the one derived from the microscopic model of $\pi \pi$ and $K \bar{K}$ correlations.

In order to demonstrate that, we show in Fig. 5 the corresponding on-shell potential matrix elements for the ${ }^{1} S_{0}$ partial wave of the $\Lambda N$ and $\Sigma N$ channels. One can see that in the case of the $\Lambda N$ system the result generated by the scalarisoscalar part of correlated $\pi \pi$ and $K \bar{K}$ exchange is similar to the one of the $\sigma$ exchange used in the Jülich $Y N$ model A.


FIG. 5. The $\sigma$-like part of the $\Lambda N$ and $\Sigma N$ on-shell potentials in the ${ }^{1} S_{0}$ partial wave. The solid lines are derived from our microscopic model of correlated $\pi \pi$ and $K \bar{K}$ exchange. The dotted lines are obtained if the dispersion-theoretical result is parametrized by $\sigma$ exchange, while the dashed lines correspond to the $\sigma$ exchange used in the Jülich $Y N$ potential $A$ [1]. 
TABLE II. Vertex coupling constants used in the new $Y N$ model that are constrained by SU(6) symmetry and corresponding cutoff masses. The assumed $\mathrm{SU}(6)$ symmetry fixes the $F /(F+D)$ ratios to $\alpha_{p s}=2 / 5, \alpha_{v}^{e}=1, \alpha_{v}^{m}=2 / 5$ [2].

\begin{tabular}{lccc}
\hline \hline Vertex & $g_{B B^{\prime} m} / \sqrt{4 \pi}$ & $f_{B B^{\prime} m} / \sqrt{4 \pi}$ & $\Lambda_{B B^{\prime} m}(\mathrm{GeV})$ \\
\hline$N N \pi$ & 3.795 & & 1.3 \\
$\Lambda \Sigma \pi$ & 2.629 & & 1.3 \\
$\Sigma \Sigma \pi$ & 3.036 & & 1.3 \\
$N \Lambda K$ & & & \\
$N \Sigma K$ & -3.944 & & 1.2 \\
$N N \omega$ & 0.759 & & 1.2 \\
$\Lambda \Lambda \omega$ & & & 1.7 \\
$\Sigma \Sigma \omega$ & 3.317 & -2.796 & 1.4 \\
$N \Lambda K^{*}$ & 2.211 & 2.796 & 1.7 \\
$N \Sigma K^{*}$ & 2.211 & & 1.2 \\
\hline \hline
\end{tabular}

In fact, correlated $\pi \pi$ exchange is marginally stronger. It is also obvious that the parametrization of the interaction generated by correlated $\pi \pi$ exchange by an effective $\sigma$ exchange, cf. the dotted line, works rather well. From the corresponding results for the on-shell $\Sigma N$ potential one can see that here the $\sigma$ exchange used in the Jülich $Y N$ model A is clearly much stronger than what one obtains from the correlated $\pi \pi$ and $K \bar{K}$ exchange. Once again the parametrization by an effective $\sigma$ exchange provides an excellent representation of the interaction strength.

\section{RESULTS AND DISCUSSION}

\section{A. Coupling constants}

In the present $Y N$ model we take into account exchange diagrams involving the well-established lowest lying pseudoscalar and vector meson SU(3) octets. Following the philosophy of the original Jülich $Y N$ potential [1] the coupling constants in the pseudoscalar sector are fixed by strict SU(6) symmetry. In any case, this is also required for being consistent with the model of correlated $\pi \pi$ and $K \bar{K}$ exchange. The cutoff masses of the form factors belonging to the $N N$ vertices are taken over from the full Bonn $N N$ potential. The cutoff masses at the strange vertices are considered as open parameters though, in practice, their values are kept as close as possible to those found for the $N N$ vertices, cf. Table II. Note that as in Ref. [1] and in line with the arguments brought forth in Ref. [2] we neglect again the contribution from $\eta$ meson exchange. In the full Bonn $N N$ model the $\eta N N$ coupling constant was set to zero. In addition phenomenological analyses [21] and also microscopic calculations, like those based on the topological chiral soliton model (extended Skyrme model with vector mesons) [22], indicate that this coupling constant should be small. Thus, the $\eta$ contribution would be completely unimportant anyway, given the pseudoscalar nature of its coupling. For the same reason the $\eta^{\prime}$ contribution is likewise not considered.
In the vector meson sector we depart from the strategy of the original Jülich $Y N$ potential. As already mentioned above, first and most importantly the contribution of the $\rho$ meson is no longer seen as resulting from the exchange of a genuine particle that belongs to the $\mathrm{SU}(3)$ vector meson octet but is identified with the strength generated by a microscopic model of correlated $\pi \pi$ and $K \bar{K}$ in the vector-isovector channel. The effective coupling constants for $\rho$ exchange in the various $Y N$ and $Y Y$ channels have been extracted and thoroughly analysed in Ref. [15]. Thereby it was found that the result from correlated exchange deviates significantly from those implied by $\mathrm{SU}(3)$ symmetry-even though SU(3) symmetry was imposed for the bare $\rho N N$ and $\rho Y Y$ couplings, cf. Ref. [15]. In view of this it is questionable whether one should invoke SU(3) symmetry for fixing the other coupling strengths of the vector-meson octet, i.e., those of the $K^{*}$ meson and of the coupling of the $\omega$ meson to the hyperons. But in absence of any better alternative we still follow this prescription for the present model. As reference values we take here the $N N \rho$ coupling constants of the full Bonn $N N$ potential [12], which were already used for the old $Y N$ model [1,2]. However, as far as the $Y Y \omega$ coupling constants are concerned now we take into account the insight gained in Ref. [23] that the $\omega$ exchange in the full Bonn $N N$ potential represents not only the genuine $\mathrm{SU}(3) \omega$ but is also an effective parametrization of additional short-range contributions from correlated $\pi-\rho$ exchange, say, that are not included explicitly in that model. Therefore, in the Bonn $N N$ model the required $N N \omega$ coupling constant is indeed much larger than what follows from the $\mathrm{SU}(3)$ relations and this large coupling constant formed also the basis for fixing the $Y Y \omega$ coupling constants of the old Jülich $Y N$ model [1,2], cf. the discussion in Sec. 2.2 of Ref. [2]. In the present model we adopt the smaller value found in Ref. [23] which is very close to the SU(3) value. This is in line with results obtained from a dispersion-theoretical analysis of the nucleon electromagnetic form factors- the inclusion of the $\pi-\rho$ continuum sizeably reduces the $\omega N N$ coupling, compare the values found in Ref. [24] with the ones in Ref. [25]. Assuming furthermore that the $\rho$ meson couples universally to the isospin current-which fixes the $F /(F+D)$ ratio $\alpha_{V}^{e}$ to $1-$ and ideal mixing for the $\phi$ and $\omega$ mesons then yields the following relation for the $\omega$ coupling constants:

$$
\begin{aligned}
& g_{\Lambda \Lambda \omega}=g_{\Sigma \Sigma \omega}=\frac{2}{3} g_{N N \omega}, f_{\Lambda \Lambda \omega}=\frac{5}{6} f_{N N \omega}-\frac{1}{2} f_{N N \rho}, \\
& f_{\Sigma \Sigma \omega}=\frac{1}{2} f_{N N \omega}+\frac{1}{2} f_{N N \rho} .
\end{aligned}
$$

For $f_{N N \omega}$ and $\mathrm{f}_{N N \rho}$ we take over the values of the full Bonn $N N$ potential. Since $f_{N N \omega}=0$ [12] it follows that $f_{\Lambda \Lambda \omega}=-f_{\Sigma \Sigma \omega}$.

The short-range contributions from correlated $\pi-\rho$ exchange were parametrized by an effective $\omega^{\prime}$ exchange in Ref. [23] with a mass of $m_{\omega^{\prime}}=1120 \mathrm{MeV}$. We follow here the same strategy but treat the coupling constants of the $\omega^{\prime}$ to the strange baryons as free parameters to be determined in a fit to the $Y N$ data.

Like the $\rho$ also the contribution of the $\sigma$ meson is computed from a microscopic model of correlated $\pi \pi$ and $K \bar{K}-$ now from the scalar-isoscalar channel. The effective coupling constants for $\sigma$ exchange in the various $Y N$ channels have been discussed in the previous section. 
TABLE III. Parameters (effective coupling strengths $G$, cutoff masses $\Lambda$ ) used in the new $Y N$ model for the effective $\omega^{\prime}, a_{0}$, and $\kappa$ exchanges. In the case of $\omega^{\prime}$ only the vector-vector component is considered. Cutoff masses in parentheses indicate that here a product of form factors of monopole type (8) is utilized instead of the standard dipole form, cf. Eq. (7). Numbers in square brackets denote corresponding values of the model where $\kappa$ exchange is replaced by a contact term, cf. text, when different.

\begin{tabular}{lcllc}
\hline \hline Channel & Exchange & $\begin{array}{c}\text { Mass } \\
(\mathrm{GeV})\end{array}$ & $G_{B B^{\prime} \rightarrow B B^{\prime}} /(4 \pi)$ & $\Lambda_{B B^{\prime} m}(\mathrm{GeV})$ \\
\hline$\Lambda N$ & $\omega^{\prime}$ & 1.12 & 5.0 & 1.65 \\
& $\kappa$ & 1.0 & $6.0[1.8]$ & $1.45[1.5]$ \\
$\Lambda N \rightarrow \Sigma N$ & $a_{0}$ & 0.983 & 2.0 & $2.0(1.8)[2.0(2.1)]$ \\
& $\kappa$ & 1.0 & $6.0[1.9]$ & $1.45(1.65)[1.5]$ \\
$\Sigma N$ & $\omega^{\prime}$ & 1.12 & 10.75 & 1.35 \\
& $a_{0}$ & 0.983 & 5.63 & $2.0(1.45)$ \\
& $\kappa$ & 1.0 & $6.0[2.4]$ & $1.65[1.5]$ \\
\hline \hline
\end{tabular}

Besides replacing the conventional $\sigma$ and $\rho$ exchanges by correlated $\pi \pi$ and $K \bar{K}$ exchange, there are in addition some other new ingredients in the present $Y N$ model. First of all, we now take into account contributions from $a_{0}(980)$ exchange. The $a_{0}$ meson is present in the original Bonn $N N$ potential [12], and for consistency should also be included in the $Y N$ model. Secondly, we consider the exchange of a strange scalar meson, the $\kappa$, with mass $\sim 1000 \mathrm{MeV}$. Let us emphasize, however, that like in the case of the $\sigma$ meson these particles are not viewed as being members of a scalar meson SU(3) multiplet, but rather as representations of strong meson-meson correlations in the scalar-isovector $(\pi \eta-K \bar{K})$ [17] and scalarisospin-1/2 $(\pi K)$ channels [16], respectively. In principle, their contributions can also be evaluated along the lines of Ref. [15], however, for simplicity in the present model they are effectively parametrized by one-boson-exchange diagrams with the appropriate quantum numbers assuming the coupling constants to be free parameters. The parameters specifying those ingredients are summarized in Table III.

Thus we have the following scenario: The long- and intermediate-range part of our new $Y N$ interaction model is completely determined by $\mathrm{SU}(6)$ constraints (for the pseudoscalar and to some extent also for the vector mesons) and by correlated $\pi \pi$ and $K \bar{K}$ exchange. The short-range part is viewed as being also due to correlated meson-meson exchanges but in practice is parametrized phenomelogically in terms of one-boson-exchange contributions in specific spin-isospin channels. In particular, no $\mathrm{SU}(3)$ relations are imposed on the short-range part. This assumption is based on our observation that the contributions in the $\rho$ exchange channel as they result from correlated $\pi \pi$ and $K \bar{K}$ no longer fulfill SU(3) relations, but it also acknowledges the fact that at present there is no general agreement about who are the actual members of the lowest-lying scalar meson SU(3) multiplet. A graphical representation of all meson-exchange contributions that are included in the new $Y N$ model is given in Fig. 6.

In recent investigations of the $N N$ interaction within the framework of chiral perturbation theory [26] only pionic
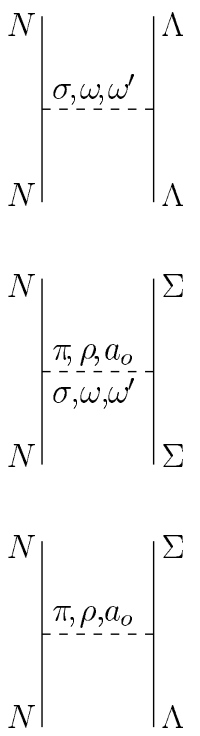

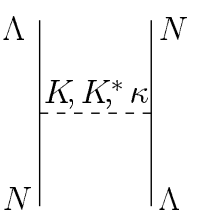

(a)



(b)



(c)

FIG. 6. Contributions to the interaction in the (a) $\Lambda N$ and (b) $\Sigma N$ channels and in the (c) $\Lambda N \rightarrow \Sigma N$ transition. Note that only $\pi, K, \omega$, and $K^{*}$ exchange are considered as being due to genuine $\mathrm{SU}(3)$ mesons. The other contributions are either fixed from correlated $\pi \pi$ and $K \bar{K}$ exchange $(\sigma, \rho)$ or are viewed as an effective parametrization of meson-meson correlations $\left(a_{0}, \kappa, \omega^{\prime}\right)$ in the corresponding spin-isospin channels.

degrees of freedom are taken into account and all short-range physics is parametrized by contact terms. This is certainly also an option that one should explore for the $Y N$ system [27] in the future [28]. As a first step we consider here an alternative model where the contributions of the $\kappa(1000)$ meson-whose mass and even existence is still under dispute [29] - are substituted by a contact term. In practice this means that we replace the product of the $\kappa$ coupling constants and propagator, $G_{B B^{\prime} \rightarrow B B^{\prime}} /\left(m_{\kappa}^{2}-t\right)$, by $G_{B B^{\prime} \rightarrow B B^{\prime}} / m_{\kappa}^{2}$ and readjust only the parameters related to the $\kappa$ exchange (with one exception). Those parameters can be found also in Table III, in square brackets, for those cases where they differ from the values of our regular model. Results corresponding to the model with the contact term will also be presented in the next section.

In the fitting procedure we only take into account data on total cross sections (and energies near the corresponding thresholds) for the channels $\Lambda p$ [30-32], $\Sigma^{-} p$ [33], $\Sigma^{-} p \rightarrow$ $\Lambda n$ [34], $\Sigma^{-} p \rightarrow \Sigma^{0} n$ [34], and $\Sigma^{+} p$ [33]. Differential cross sections but also total cross sections at higher energies [35-37] are therefore genuine predictions of our model. As already mentioned above, the free parameters in our model consist of the cut-off masses at the strange vertices and the coupling constants of the $a_{0}(980), \kappa(1000)$, and $\omega^{\prime}(1120)$ mesons. When adjusting those parameters to the empirical data it turned out that the results are not very sensitive to the cut-off masses in the pseudoscalar sector and we fixed them to be close to the cutoff mass used at the $\pi N N$ vertex. There is also only a weak sensitivity to the cut-off masses used for the correlated $\pi \pi-K \bar{K}$ contributions in the $\sigma$ and $\rho$ channels. This is due to the chosen analytic form of the form factors that practically 
does not change the strength of the corresponding potentials as they result from the microscopic model-which is of course intended, cf. the discussion in Sec. III. Besides the cut-off masses of the vector mesons we found that also the parameters of the $a_{0}(980)$ and $\kappa(1000)$ mesons, viewed here as effective parametrization of correlated $\pi \eta$ and $\pi K$ exchange, have a sizable influence. In fact, without the contributions of the latter two mesons we would not have been able to achieve a satisfactory description of the data. Note that those two exchanges were not considered in the original Jülich model [1]. We should say that values of the coupling strengths and cut-off masses for those scalar mesons are strongly correlated and cannot be fixed independently from a fit to the data. Thus, one should not attribute any physical significance to the actual values of the coupling strengths or cut-off masses that we found individually.

Finally we want to mention that the fit to the available $Y N$ data did not constrain the relative magnitude of the ${ }^{1} S_{0}$ and ${ }^{3} S_{1}$ partial waves in the $\Lambda N$ system. Thus, as a further constraint, we required the ${ }^{1} S_{0}$ scattering length to be larger than the one for ${ }^{3} S_{1}$ - as it seems to be necessary if one wants to achieve a bound hypertriton [38]. A first application of the new $Y N$ model in three-body calculations confirmed that it yields indeed a bound hypertriton state [39].

\section{B. The scattering equation}

The original $Y N$ model of the Jülich group was derived within the framework of time ordered perturbation theory (TOPT) [1]. In this approach retardation effects from the meson-exchange diagrams are retained (and those of baryonexchange as well) and as a consequence the interaction depends explicitly on the starting energy. This is not convenient if one wants to apply the $Y N$ model in conventional few-body [40-47] or many-body [48-51] investigations. Thus, in Ref. [2] the Jülich group presented energy-independent versions of their $Y N$ model where the energy dependence was removed in such a way that basically all other characteristics of the original model could be kept. The detailed comparison of the TOPT model and its energy-independent counterpart performed in Ref. [2] made clear that this goal was indeed achieved.

Since we are also interested to facilitate an application of our new $Y N$ model in future few- and many-body investigations we will likewise present here an energy-independent interaction. This implies that we do not use the (relativistic) TOPT scattering equation of Ref. [1] but instead solve the nonrelativistic (coupled-channel) Lippmann-Schwinger equation

$$
T_{i, j}=V_{i, j}+\sum_{k} V_{i, k} G_{k} T_{k, j}
$$

to obtain the scattering amplitude $T_{i, j}$. Here the indices $(i, j, k)$ stand for the $\Lambda N$ and $\Sigma N$ channels and the nonrelativistic Green's function $G_{k}$ is given by

$$
G_{k}=\left[\frac{q_{k}^{2}-\mathbf{q}^{\prime 2}}{2 \mu_{k}}+i \varepsilon\right]^{-1},
$$

where $\mu_{k}=M_{Y} M_{N} /\left(M_{Y}+M_{N}\right)$ is the reduced mass and $\mathbf{q}^{\prime}$ the c.m. momentum in the intermediate $Y N$ channel. $q_{k}=q_{k}(z)$ denotes the on-shell momentum in the intermediate $Y N$ state defined by $z=\sqrt{M_{Y}^{2}+q_{k}^{2}}+\sqrt{M_{N}^{2}+q_{k}^{2}}$. The latter equation guarantees that the $\Sigma N$ channel opens exactly at the physical threshold. Note that $q_{\Sigma N}$ is imaginary for starting energies below the $\Sigma N$ threshold $\left(z<M_{\Sigma}+M_{N}\right)$. Explicit expressions for the potential matrix elements $V_{i, j}$ for the various exchange diagrams can be found in Ref. [1]. The dependence on the starting energy $z$ is removed via the prescriptions given in Eq. (4.7) of Ref. [2]. Note that the potential matrix elements $V_{i, j}$ are derived by assuming isospin symmetry. However, the Lippmann-Schwinger equation (14) is solved in particle space using the proper physical masses of the baryons for the various $\Sigma N$ channels. Furthermore, in the charged channels the Coulomb potential is taken into account. Since we solve the Lippmann-Schwinger equation in momentum space this is done by means of the Vincent-Phatak method $[1,52]$.

\section{Hyperon-nucleon observables}

In Fig. 7 we compare the integrated cross sections obtained from the new $Y N$ potential (solid curves) with the $Y N \rightarrow$ $Y^{\prime} N$ scattering data. Obviously, a good reproduction of the empirical data [30-34] is achieved. Also shown are results from the original Jülich $Y N$ model A [1] (dash-dotted curves). The main qualitative differences between the two models appear in the $\Lambda p \rightarrow \Lambda p$ channel, for which the Jülich model [1] (with standard $\sigma$ and $\rho$ exchange) predicts a broad shoulder at $p_{\text {lab }} \approx 350 \mathrm{MeV} / c$. This structure, which is not supported by the available experimental evidence, is due to a bound state in the ${ }^{1} S_{0}$ partial wave of the $\Sigma N$ channel. It is not present in the new model anymore. (We should say, however, that the new model has a bound state, too. But with a binding energy of about $400 \mathrm{MeV}$ below the $\Lambda N$ threshold it is located completely outside of the physical region. One could speculate, of course, that this bound state is a manifestation of the Pauli forbidden (11)s state at the quark level [53].) Furthermore, the cusp structure at the opening of the $\Sigma N$ threshold is much less pronounced in the new model. In the old model this structure was primarily caused by a large amplitude in the tensor-coupled ${ }^{3} S_{1}-{ }^{3} D_{1}$ partial wave of the $\Lambda N-\Sigma N$ transition. This amplitude is now much smaller. As a consequence also the transition cross section for $\Sigma^{-} p \rightarrow \Lambda n$ is now somewhat smaller, though still in line with the empirical informations. In the $\Sigma^{-} p$ channel the new model yields a stronger energy dependence of the reaction cross section as it is favored by the available cross-section data. In the other two measured reaction channels the agreement with the data is equally good, if not better, for the new model.

Note that the $\Sigma^{+} p$ and $\Sigma^{-} p$ elastic cross sections are not "true" total cross sections. The cross sections that were measured are defined as [33]

$$
\sigma=\frac{2}{\cos \theta_{\max }-\cos \theta_{\min }} \int_{\cos \theta_{\min }}^{\cos \theta_{\max }} \frac{d \sigma(\theta)}{d \cos \theta} d \cos \theta,
$$

with typical values -0.2 to -0.5 for $\cos \theta_{\min }$ and 0.3 to 0.5 for $\cos \theta_{\max }$. [In order to stay as close as possible to the plotted experimental data, the theoretical curves in Figs. 9(c) and (d) have been calculated with $\cos \theta_{\min }=-0.5$ and $\cos \theta_{\max }=0.5$.] 

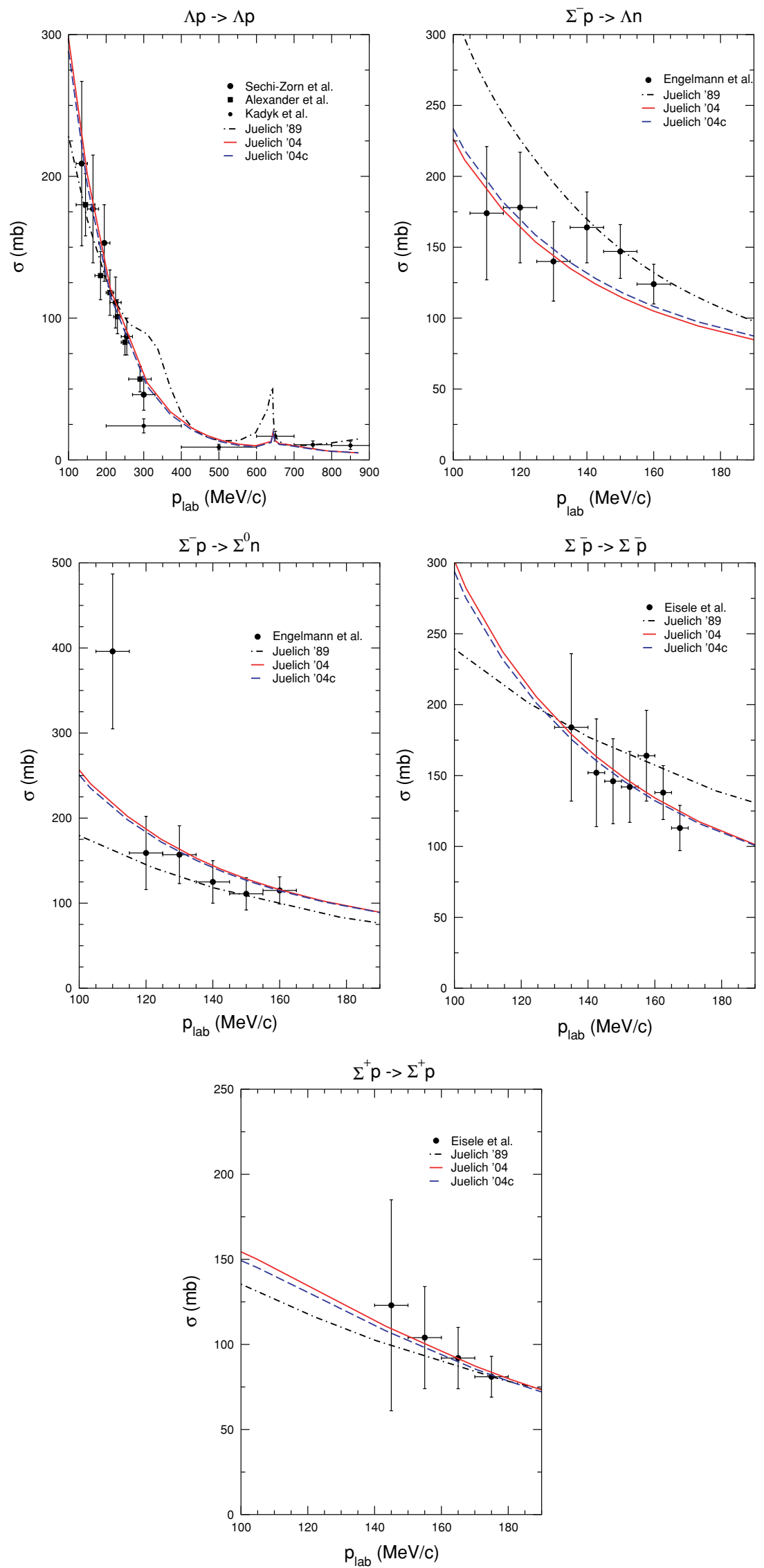

FIG. 7. (Color online) Total $Y N$ scattering cross sections as a function of the laboratory momentum. The solid lines are results of the new $Y N$ model, based on correlated $\pi \pi$ and $K \bar{K}$ exchange, while the dash-dotted lines are results of the Jülich $Y N$ model A [1]. The dashed lines are results of an alternative model where $\kappa$ exchange is replaced by a contact term, cf. text. The data are from Refs. [30-34]. 

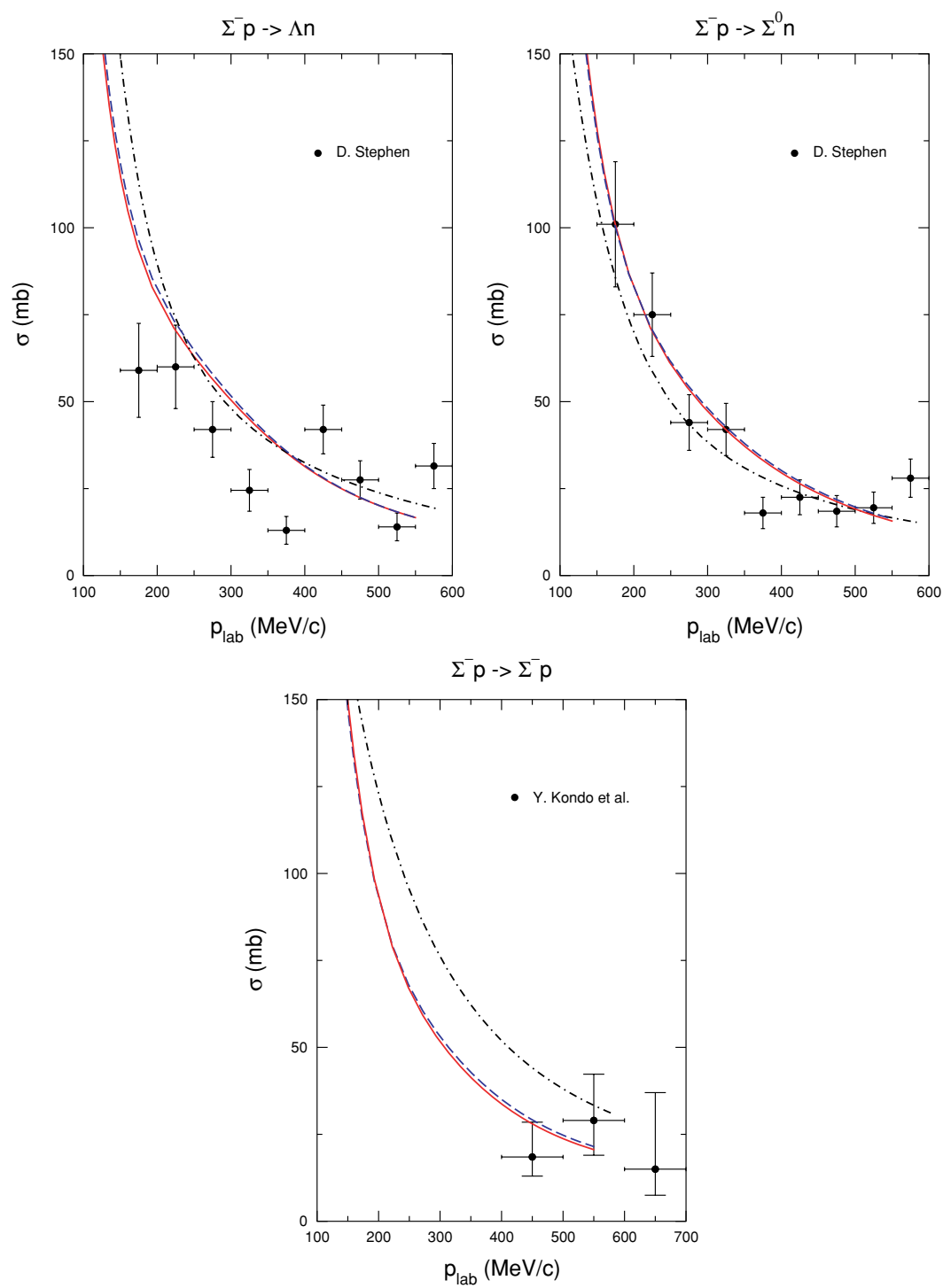

Cross sections at somewhat higher energies are presented in Fig. 8. Note that the data shown in this figure have not been taken into account in the fitting process and therefore the results are genuine predictions of the model. Also here the agreement with the data is satisfactory.

The differential $Y N$ scattering cross sections presented in Fig. 9 are likewise genuine predictions of our $Y N$ model. We want to point out that the empirical information in those figures comes from data taken from a finite momentum interval, e.g., $160<p_{\text {lab }}<180 \mathrm{MeV} / c$ for the $\Sigma^{+} p$ channel [30], whereas the calculations were performed for the central value of that momentum interval as it is given in the various plots. Note also that the original $Y N$ model of the Jülich group was fitted to the data without including the Coulomb interaction (and without taking into account the mass splitting between $\Sigma^{-}, \Sigma^{0}$, and $\left.\Sigma^{+}\right)$. Thus, the corresponding results presented in Fig. 9 do not show the strong forward peak caused by the Coulomb amplitude in the charged channels.

Evidently, the data on differential cross sections are also rather well reproduced by our new $Y N$ model. In comparison to the results of the original Jülich model one can say that the
FIG. 8. (Color online) Total $Y N$ scattering cross sections as a function of the laboratory momentum. Comparison with data at higher energies. Same description of curves as in Fig. 7. The data are from Refs. [35,36]. angular dependence in the $\Sigma^{-} p$ channel is now much better described and it seems to be more in line with the trend of the angular dependence exhibited by the data in the $\Sigma^{-} p \rightarrow \Lambda n$ channel too.

The dashed curves in Figs. 7, 8, and 9 are results from an alternative model where the contributions from the disputed $\kappa(1000)$ meson have been replaced by a contact interaction. Obviously there is practically no sensitivity to the concrete range of the contribution in the scalar channel with isospin $1 / 2$-besides that it has to be of fairly short range. In this context we want to mention that we could achieve a comparable description of the data even with a $\kappa$ mass as low as $800 \mathrm{MeV}$ [54].

For exploring the differences between the original Jülich $Y N$ model and our new model in more detail we present in Figs. 10 and 11 further observables where, however, no data are available. Figure 10 contains differential cross sections, polarizations, and the depolarization parameter $D_{n n}$ (definition and explicit expressions for those observables can be found in the Appendix B of Ref. [2]) for the $\Lambda N$ channel. We present predictions at two energies, one $\left(p_{\text {lab }}=150 \mathrm{MeV} / c\right)$ close to 

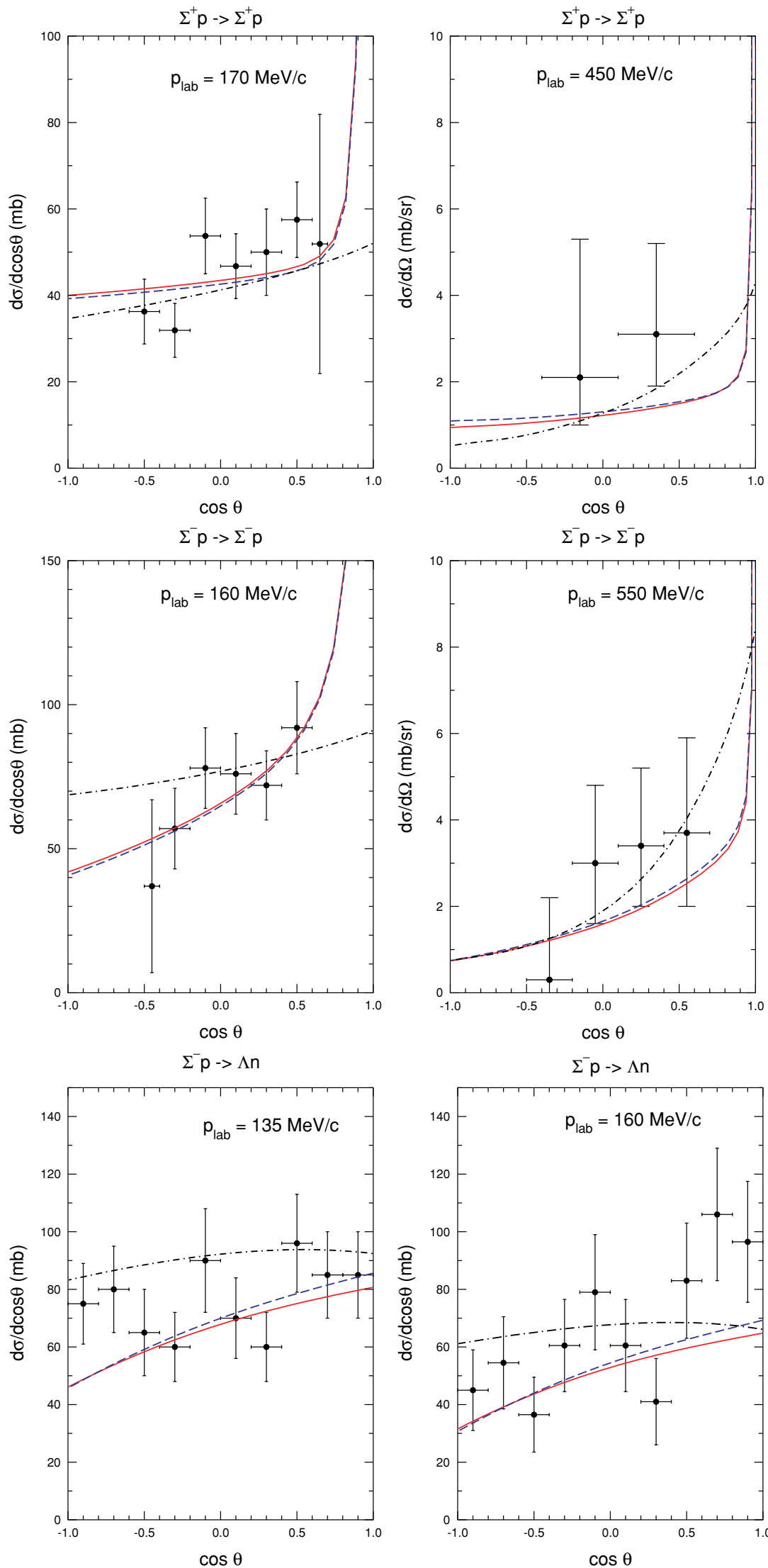

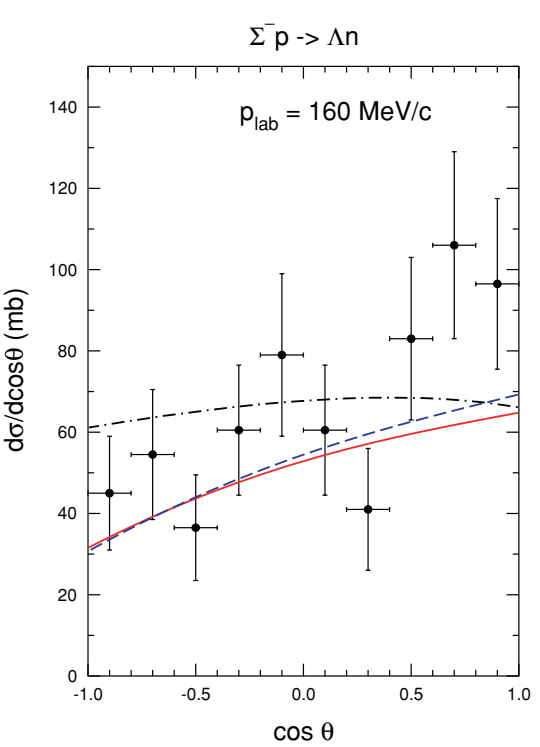

FIG. 9. (Color online) Differential $Y N$ scattering cross sections at selected laboratory momenta where data are available. Same description of curves as in Fig. 7. The data are from Refs. [33,34,36,37]. 
TABLE IV. YN low energy parameters in the ${ }^{1} S_{0}$ and ${ }^{3} S_{1}$ partial waves derived from our new model (J04) together with the corresponding results of the Jülich model A [1]. J04c refers to results of an alternative model where $\kappa$ exchange is replaced by a contact term, cf. text.

\begin{tabular}{|c|c|c|c|c|c|}
\hline Channel & Model & $a_{s}(\mathrm{fm})$ & $r_{s}(\mathrm{fm})$ & $a_{t}(\mathrm{fm})$ & $r_{t}(\mathrm{fm})$ \\
\hline \multirow[t]{3}{*}{$\Lambda N$} & J04 & -2.56 & 2.75 & -1.66 & 2.93 \\
\hline & $\mathrm{J} 04 \mathrm{c}$ & -2.66 & 2.67 & -1.57 & 3.08 \\
\hline & A [1] & -1.56 & 1.43 & -1.59 & 3.16 \\
\hline \multirow{3}{*}{$\Sigma N(I=1 / 2)$} & J04 & $0.90-i 0.13$ & $-4.38-i 2.07$ & $-3.83-i 3.01$ & $2.79-i 0.57$ \\
\hline & $\mathrm{J} 04 \mathrm{c}$ & $0.90-i 0.13$ & $-4.29-i 2.05$ & $-3.63-i 3.09$ & $2.78-i 0.60$ \\
\hline & A [1] & $1.42-i 0.08$ & $-0.49-i 0.27$ & $2.47-i 3.74$ & $1.61-i 0.64$ \\
\hline \multirow[t]{3}{*}{$\Sigma N(I=3 / 2)$} & J04 & -4.71 & 3.31 & 0.29 & -11.54 \\
\hline & $\mathrm{J} 04 \mathrm{c}$ & -4.58 & 3.32 & 0.28 & -11.63 \\
\hline & A [1] & -2.26 & 5.22 & -0.76 & 0.79 \\
\hline
\end{tabular}

the $\Lambda N$ threshold and one $\left(p_{\text {lab }}=600 \mathrm{MeV} / c\right)$ close to (but below) the $\Sigma N$ threshold. The results at the higher energy reveal that the new model differs drastically from the old one. The differential cross section in the new model is strongly forward-peaked whereas the one of the old models peaks in forward and backward direction. The polarization and $D_{n n}$ have even different signs. The observables at the lower energy are still dominated by the $S$ waves and therefore exhibit only minor differences. But one can see from the differential cross section that the onset of higher partial waves occurs earlier for the new $Y N$ model.

Similarly striking differences are present also in the predictions for other differential observables though we refrain from showing them here.

For the various $\Sigma N$ channels we present predictions for the polarization and the depolarization parameter $D_{n n}$ at $p_{\text {lab }}=$ $500 \mathrm{MeV} / c$, cf. Fig. 11. Also here one can see that, in general, there are large differences between the results of the old and the new model.

\section{Low energy parameters and phase shifts}

For the computation of the low energy parameters and phase shifts we omit the Coulomb interaction and ignore the mass differences between the $\Sigma$ 's and proton and neutron so that we can solve the Lippmann-Schwinger equation in isospin basis. This allows us to present also results for the $\Sigma N$ system in the $I=1 / 2$ channel. The $Y N$ S-wave low energy parameters are listed in Table IV while phase shifts for selected partial waves are shown in Figs. 12 and 13.

From Table IV one can see that the scattering lengths in the ${ }^{3} S_{1} \Lambda N$ partial wave $\left(a_{t}\right)$ are of similar magnitude for the old and new $Y N$ models, but in the ${ }^{1} S_{0}$ state $\left(a_{s}\right)$ the new model yields a significantly larger value. The stronger ${ }^{1} S_{0}$ component of the new model is reflected in the larger $\Lambda p$ cross section near threshold, cf. Fig. 7, and it is expected to provide sufficient strength in order to support a bound hypertriton state [39]. Indeed recent $Y N$ models like NSC97f of the Nijmegen group [6] or the Ehime model 00A [7], that apparently lead to a bound hypertriton [7,55], predict singlet scattering lengths that are very similar to that of our new model.
In this context we want to mention that the static version of the old Jülich $Y N$ model [2] did not support a hypertriton bound state [40]. However, in that model both the ${ }^{1} S_{0}$ as well as the ${ }^{3} S_{1} \Lambda N$ scattering lengths are considerably smaller [2] than in our new $Y N$ model.

The scattering lengths and effective ranges for $\Sigma N$ with $I=1 / 2$ are complex because this channel is coupled to the $\Lambda N$ system. In the singlet case the scattering lengths are comparable for the two models whereas in the triplet case they even have opposite signs. We want to emphasize, however, that in both models the latter partial wave is attractive. But in the original Jülich model the attraction is so strong that there is a near-threshold quasibound state in the $\Sigma N$ channel that causes the real part of $a_{t}$ to be positive-like in case of the corresponding $N N$ partial wave and the deuteron. Let us mention that practically the same situation occurs in the Nijmegen model NSC97f, whose pole structure has been investigated and thoroughly discussed in Ref. [56]. As a consequence of the near-threshold pole both these models yield a very pronounced cusplike structure in the $\Lambda p$ cross section at the opening of the $\Sigma N$ channel, cf. Figs. 7 and 2 in Ref. [6], respectively. In our new $Y N$ model, on the other hand, the cusp at the $\Sigma N$ threshold is much less pronounced. Note that the ${ }^{1} S_{0}$ partial wave is attractive too. As already mentioned above, in the original Jülich model there is a bound state in the $\Sigma N$ channel-as evidenced by the broad bump in the $\Lambda p$ cross section around $p_{\text {lab }} \approx 350 \mathrm{MeV} / c$. And the new $Y N$ model has also a bound state which is located, however, around $400 \mathrm{MeV}$ below the $\Lambda N$ threshold and therefore completely outside of the physically relevant region.

Let us finally come to the $\Sigma N$ channel with $I=3 / 2$. Here we see that the singlet scattering length of the new model is about twice as large as the one of the original Jülich model. Note that a comparably large singlet scattering length is also predicted by all of the $Y N$ models presented in Ref. [6]. The scattering lengths for ${ }^{3} S_{1}$ are small in both cases, but of opposite sign. Now, however, it is indeed so that our new $Y N$ model is repulsive in this partial wave whereas the old model is attractive. It is interesting that basically all available $Y N$ models predict rather small values for the spin-triplet scattering length of the $\Sigma N I=3 / 2$ channel [1,4-6,11], though there is no general trend as far as the sign is concerned. We also observe 



$\Lambda p->\Lambda p$
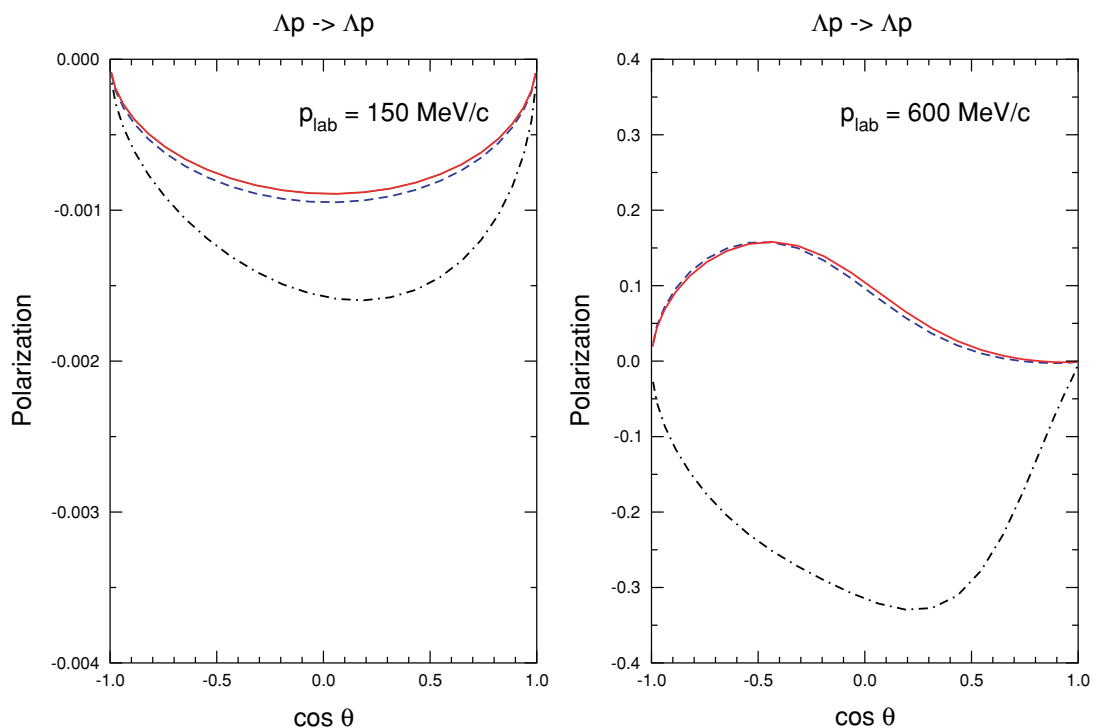

$\Lambda \mathrm{p}->\mathrm{p}$

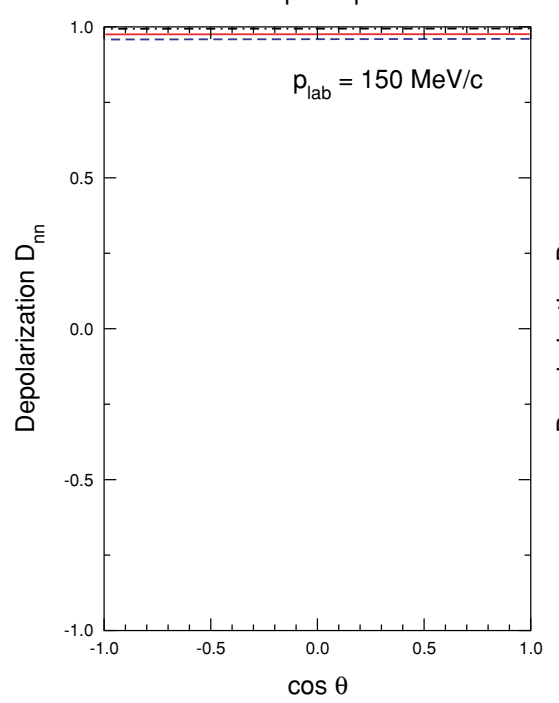

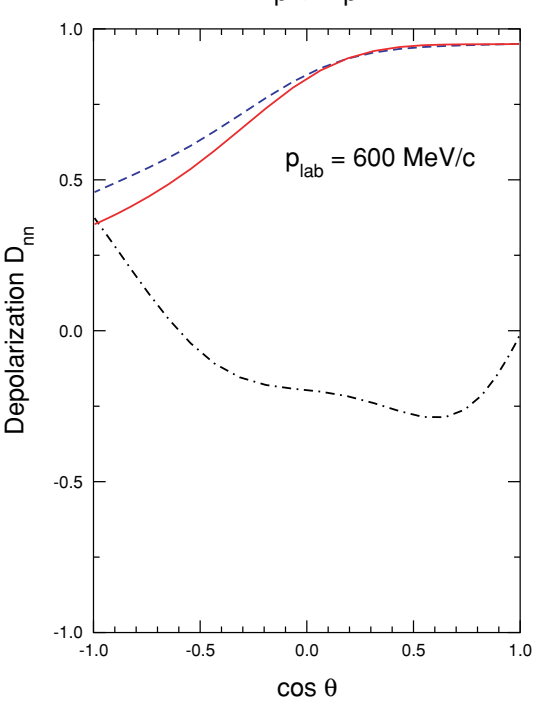

$\Lambda \mathrm{p} \rightarrow \Lambda \mathrm{p}$
FIG. 10. (Color online) Some differential observables for the reaction $\Lambda N$ at the laboratory momenta 150 and $600 \mathrm{MeV} / c$. Same description of curves as in Fig. 7. 

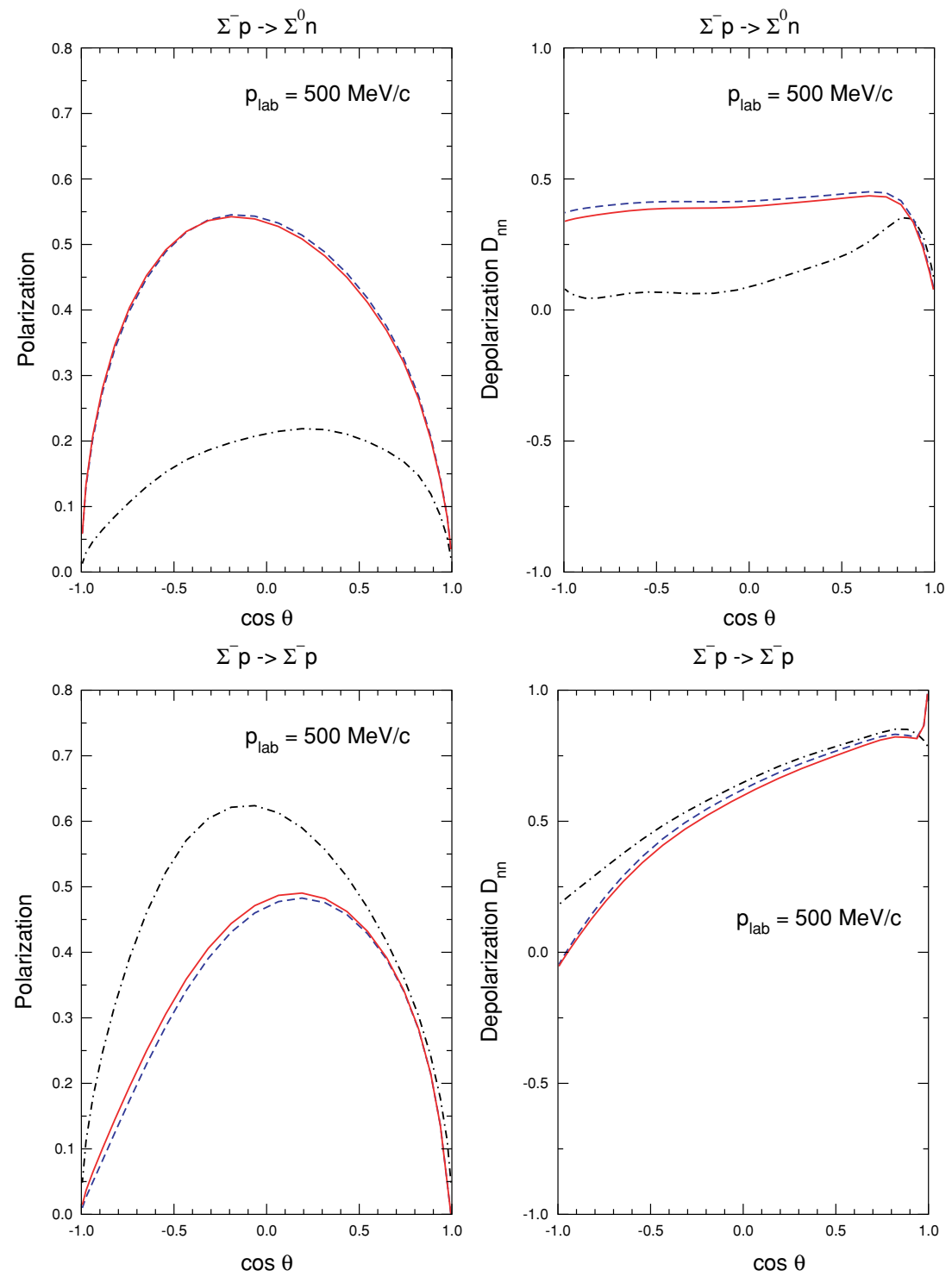

FIG. 11. (Color online) Differential cross sections and polarizations for the reactions $\Sigma N$ at the laboratory momentum $500 \mathrm{MeV} / c$. Same description of curves as in Fig. 7.




FIG. 12. (Color online) Phase shifts for selected $\Lambda N$ and $\Sigma N S$ and $D$ waves as a function of the laboratory energy. Same description of curves as in Fig. 7.


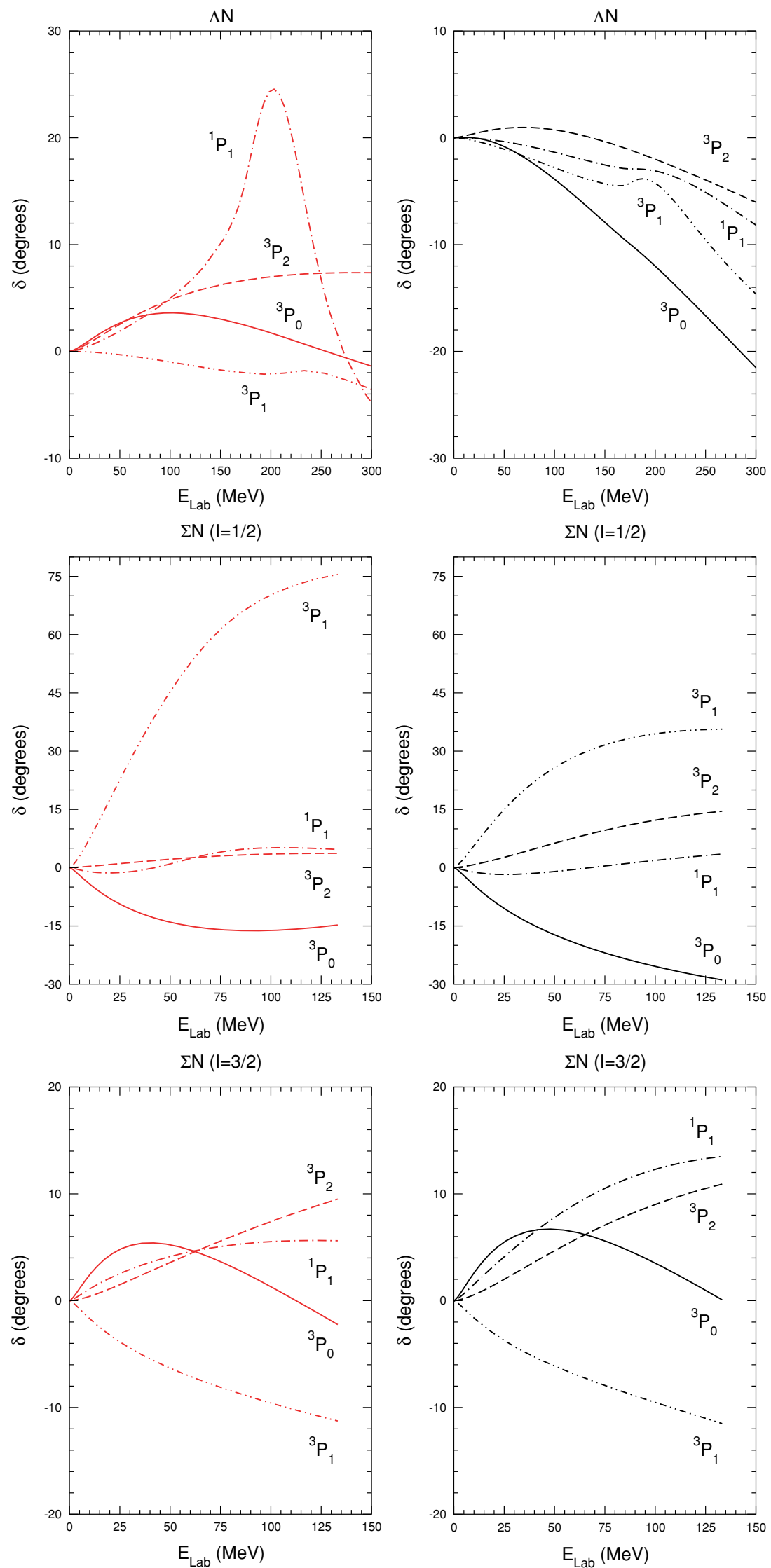

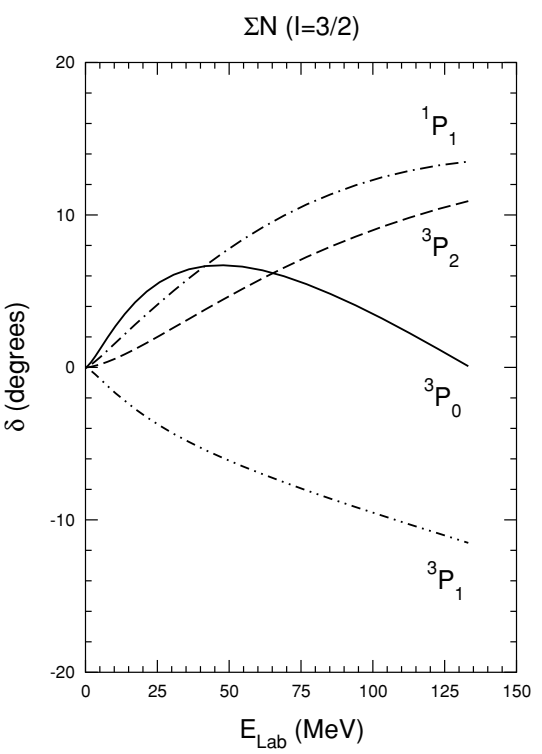

FIG. 13. (Color online) Phase shifts for $\Lambda N$ and $\Sigma N P$ waves as a function of the laboratory energy. The left panel shows results of our new $Y N$ model and the right panel those of the original Jülich $Y N$ model $A$ [1]. 
an unnaturally large value for the triplet effective range, which is clearly related to the strong suppression of the corresponding scattering length. Such a scenario will require special attention when this channel is considered in effective field theory (for further discussion, see Sec. V).

Predictions for $\Lambda N$ and $\Sigma N$ phase shifts for selected $S$ and $D$-waves are shown in Fig. 12 and those for $P$-waves can be found in Fig. 13. The $\Sigma N S$-wave phase shifts reflect the features that we already discussed in the context of the scattering lengths. For example one can see that the phase shift for the ${ }^{3} S_{1} I=1 / 2$ state starts at $180^{\circ}$ for the original Jülich model, as it is expected for a partial wave where a bound state is present. For the $I=3 / 2$ state the corresponding phase is positive, reflecting an attractive interaction, whereas the phase shift resulting from the new $Y N$ model is negative. Note that the phases for ${ }^{1} S_{0}$ and $I=1 / 2$ should both start at $180^{\circ}$ because, as mentioned above, there is a bound state in both models.

The opening of the $\Sigma N$ channel at around $E_{\text {lab }} \approx 170 \mathrm{MeV}$ is cleary reflected in the ${ }^{3} S_{1}$ phase shift of the $\Lambda N$ system. But its effect on the ${ }^{3} D_{1}$ phase shift is even more striking where, for the old Jülich model, the phase even goes through $90^{\circ}$. In fact, the resonance-like behavior in that partial wave is predominantly responsible for the strong enhancement of the $\Lambda N$ cross section in the vicinity of the $\Sigma N$ threshold, cf. Fig. 7. In addition, the transition amplitude ${ }^{3} D_{1}(\Lambda N) \leftrightarrow$ ${ }^{3} S_{1}(\Sigma N)$ provides a significant contribution to the $\Sigma^{-} p \rightarrow$ $\Lambda n$ cross section. In the new model the ${ }^{3} D_{1}$ phase shift of the $\Lambda N$ system is much smaller. Accordingly, the cusplike structure at the $\Sigma N$ threshold is much less pronounced and the $\Sigma^{-} p \rightarrow \Lambda n$ cross section is somewhat reduced in this model, as can be seen in Fig. 7 .

The predictions for the $P$ waves (Fig. 13) show a varying picture. In the $\Lambda N$ system most of the phases are now attractive whereas they are mostly repulsive for the old model. This concerns in particular the ${ }^{1} P_{1}$ amplitude, which is fairly large in the new model, but also the ${ }^{3} P_{0}$ partial wave. In the $I=3 / 2$ channel of the $\Sigma N$ system the results of the two models are qualitatively rather similar. To some extent this is also the case for the $I=1 / 2$ channel though here the ${ }^{3} P_{1}$ amplitude of the new $Y N$ model is significantly larger than the one of the old Jülich model. Indeed the simultaneous enhancement in the ${ }^{3} P_{1}(\Sigma N)$ and ${ }^{1} P_{1}(\Lambda N)$ phase shifts is caused by a stronger antisymmetric spin-orbit force between the $\Lambda N$ and $\Sigma N$ channels in the new model. The increase is primarily due to the $\rho$ exchange contribution whose strength for the $\Lambda N \rightarrow$ $\Sigma N$ transition, fixed from correlated $\pi \pi-K \bar{K}$ exchange, is about twice as large as what was used in the old Jülich model, cf. Table 11 of Ref. [15]. In this context let us mention that some other $Y N$ models exhibit a similarly strong coupling between those partial waves and channels [57].

\section{SUMMARY AND OUTLOOK}

We have presented a meson-exchange model of the $Y N$ interaction where - as the main new feature - the contributions both in the scalar-isoscalar $(\sigma)$ and the vector-isovector $(\rho)$ channels are constrained by a microscopic model of correlated $\pi \pi$ and $K \bar{K}$ exchange.
An essential part of baryon-baryon interactions is the strong medium-range attraction, which in one-boson-exchange models is parametrized by exchange of a fictitious scalarisoscalar meson with mass around $500 \mathrm{MeV}$. In extended meson exchange models this part is naturally generated by two-pion exchange contributions. As well as uncorrelated two-pion exchange, correlated contributions must be included in which the exchanged pions interact during their exchange; these terms in fact provide the main contribution to the intermediate-range interaction.

As kaon exchange is an essential part of hyperon-nucleon interactions a simultaneous investigation of correlated $\pi \pi$ and $K \bar{K}$ exchanges is clearly necessary. In Ref. [15] the correlated $\pi \pi$ and $K \bar{K}$ exchange contributions in various baryon-baryon channels have therefore been investigated within a microscopic model for the transition amplitudes of the baryon-antibaryon system $\left(B \overline{B^{\prime}}\right)$ into $\pi \pi$ and $K \bar{K}$ for energies below the $B \overline{B^{\prime}}$ threshold. The correlations between the two mesons have been taken into account by means of $\pi \pi-K \bar{K}$ amplitudes, determined in the field theoretical framework of Refs. [16-18], which provide an excellent description of empirical $\pi \pi$ data up to $1.3 \mathrm{GeV}$. With the help of unitarity and dispersion-theoretical methods, the baryonbaryon amplitudes for correlated $\pi \pi$ and $K \bar{K}$ exchange in the $J^{P}=0^{+}(\sigma)$ and $J^{P}=1^{-}(\rho) t$-channels have then been determined. With this model it is possible to reliably take into account correlated $\pi \pi$ and $K \bar{K}$ exchange in both the $\sigma$ and $\rho$ channels for various baryon-baryon reactions. Given the strong constraints on $\sigma$ as well as $\rho$ exchange from correlated $\pi \pi$ exchange, a more sound microscopic model for the $Y N$ interaction can hence now be constructed.

Besides contributions from correlated $\pi \pi$ and $K \bar{K}$ exchange the present model incorporates also the standard one-boson exchanges of the lowest pseudoscalar and vector meson multiplets with coupling constants fixed by SU(6) symmetry relations. Thus, in the present model the long- and intermediate-range part of the $Y N$ interaction is completely determined - either by SU(6) constraints or by correlated $\pi \pi$ and $K \bar{K}$ exchange.

In addition there are some short-ranged ingredients. First of all, the contribution from the $a_{0}(980)$ meson is taken into account. Secondly, we consider the exchange of a strange scalar meson, the $\kappa$, with mass $\sim 1000 \mathrm{MeV}$. (Note that these pieces were not taken into account in the earlier $Y N$ models of the Jülich group [1,2].) These short-ranged contributions are also viewed as being due to correlated meson-meson exchanges but in practice they are parametrized phenomelogically in terms of one-boson-exchange contributions in the corresponding spin-isospin channels. In particular, no SU(3) relations are imposed on the short-range part. This assumption is based on our observation that the contributions in the $\rho$ exchange channel as they result from correlated $\pi \pi$ and $K \bar{K}$ no longer fulfill SU(3) relations, but it also acknowledges the fact that at present there is no general agreement about who are the actual members of the lowest-lying scalar meson SU(3) multiplet.

The new $Y N$ model provides a rather satisfactory reproduction of the available $Y N$ data. It describes not only the integrated cross sections for $\Lambda p$ and the various $\Sigma N$ channels but also the 
few available data on differential cross sections, even though the latter were not included in the fitting procedure. We see that as an indication that the data are compatible with the assumption of SU(6) symmetry for the pseudoscalar sector of our $Y N$ model.

As the main qualitative difference between the old $Y N$ Jülich model [1] (with standard $\sigma$ and $\rho$ exchange) we want to mention that the broad shoulder at $p_{\text {lab }} \approx 350 \mathrm{MeV} / c$ in the $\Lambda p \rightarrow \Lambda p$ channel, predicted by that model but not seen in the experiments, is no longer present in the new model. But, as a more detailed comparison revealed, there are also striking differences between these two models in the predictions for the individual partial waves. For example, in the new model the triplet $S$ wave in the $I=3 / 2$ channel of the $\Sigma N$ system is repulsive and some of the $P$-wave amplitudes are significantly larger. Thus, it will be interesting to see the performance of the new $Y N$ interaction model in applications to few- and many-body systems involving hyperons [39].

This study also paves the way for a systematic investigation in the framework of effective field theory, see Ref. [28]. In such a framework, pion- and kaon exchange supplemented by fourbaryon contact interactions (these encode the contributions from the exchange of heavier mesons not linked to chiral symmtery) is considered to generate a potential based on the power counting rules. It remains to be seen how well such a more systematic approach can indeed describe the data and what conclusions can be drawn about three-baryon forces that naturally arise in such a framework.

\section{ACKNOWLEDGMENTS}

We thank J. Speth and W. Melnitchouk for collaboration during the early stages of this investigation. We also thank A. Nogga for a careful reading of our manuscript. This research is part of the EU Integrated Infrastructure Initiative Hadron Physics Project under Contract No. RII3-CT-2004-506078. The work was supported in part by DFG through funds provided to the special research grant TR-16 "Subnuclear Structure of Matter."
[1] B. Holzenkamp, K. Holinde, and J. Speth, Nucl. Phys. A500, 485 (1989).

[2] A. Reuber, K. Holinde, and J. Speth, Nucl. Phys. A570, 543 (1994).

[3] M. M. Nagels, T. A. Rijken, and J. J. de Swart, Phys. Rev. D 15, 2547 (1977).

[4] M. M. Nagels, T. A. Rijken, and J. J. de Swart, Phys. Rev. D 20, 1633 (1979).

[5] P. M. M. Maessen, T. A. Rijken, and J. J. de Swart, Phys. Rev. C 40, 2226 (1989).

[6] Th. A. Rijken, V. G. Stoks, and Y. Yamamoto, Phys. Rev. C 59, 21 (1999).

[7] K. Tominaga and T. Ueda, Nucl. Phys. A693, 731 (2001).

[8] U. Straub et al., Nucl. Phys. A483, 686 (1988); A508, 385c (1990).

[9] Z. Y. Zhang, A. Faessler, U. Straub, and L. Ya. Glozman, Nucl. Phys. A578, 573 (1994).

[10] Z. Y. Zhang, Y. W. Yu, P. N. Shen, L. R. Dai, A. Faessler, and U. Straub, Nucl. Phys. A625, 59 (1997).

[11] Y. Fujiwara, T. Fujita, M. Kohno, C. Nakamoto, and Y. Suzuki, nucl-th/0101014; Y. Fujiwara, C. Nakamoto, Y. Suzuki, M. Kohno, and K. Miyagawa, Prog. Theor. Phys. Suppl. 156, 17 (2004).

[12] R. Machleidt, K. Holinde, and Ch. Elster, Phys. Rep. 149, 1 (1987).

[13] R. L. Jaffe, Phys. Rev. Lett. 38, 195 (1977); 38, 617(E) (1977).

[14] H.-C. Kim, J. W. Durso, and K. Holinde, Phys. Rev. C 49, 2355 (1994).

[15] A. Reuber, K. Holinde, H.-C. Kim, and J. Speth, Nucl. Phys. A608, 243 (1996).

[16] D. Lohse, J. W. Durso, K. Holinde, and J. Speth, Nucl. Phys. A516, 513 (1990).

[17] G. Janssen, B. C. Pearce, K. Holinde, and J. Speth, Phys. Rev. D 52, 2690 (1995) [arXiv:nucl-th/9411021].

[18] C. Schütz, K. Holinde, J. Speth, B. C. Pearce, and J. W. Durso, Phys. Rev. C 51, 1374 (1995).
[19] G. Höhler, F. Kaiser, R. Koch, and E. Pietarinen, "Handbook of Pion-Nucleon Scattering" 4, Physics Data 12-1, Fachinformationszentrum, Karlsruhe, 1979.

[20] J. Haidenbauer, W. Melnitchouk, and J. Speth, nucl-th/9805014; J. Haidenbauer, W. Melnitchouk, and J. Speth, Nucl. Phys. A663, 549 c (2000).

[21] W. Grein and P. Kroll, Nucl. Phys. A338, 332 (1980); A377, 505 (1982).

[22] Ulf-G. Meißner, N. Kaiser, H. Weigel, and J. Schechter, Phys. Rev. D 39, 1956 (1989).

[23] G. Janssen, K. Holinde, and J. Speth, Phys. Rev. C 54, 2218 (1996) [arXiv:nucl-th/9508022].

[24] P. Mergell, U.-G. Meißner, and D. Drechsel, Nucl. Phys. A596, 367 (1996); H.-W. Hammer, U.-G. Meißner, and D. Drechsel, Phys. Lett. B385, 343 (1996); H.-W. Hammer and U.-G. Meißner, Eur. Phys. J. A 20, 469 (2004) .

[25] U.-G. Meißner, V. Mull, J. Speth, and J. W. van Orden, Phys. Lett. B408, 381 (1997).

[26] E. Epelbaum, W. Glöckle, and U.-G. Meißner, Nucl. Phys. A747, $362(2005)$

[27] C. L. Korpa, A. E. L. Dieperink, and R. G. E. Timmermans, Phys. Rev. C 65, 015208 (2002).

[28] H. Polinder et al., in preparation.

[29] S. Eidelman et al., Phys. Lett. B592, 1 (2004).

[30] G. Alexander et al., Phys. Rev. 173, 1452 (1968).

[31] B. Sechi-Zorn et al., Phys. Rev. 175, 1735 (1968).

[32] J. A. Kadyk et al., Nucl. Phys. B27, 13 (1971).

[33] F. Eisele et al., Phys. Lett. 37B, 204 (1971).

[34] R. Engelmann et al., Phys. Lett. 21, 587 (1966).

[35] D. Stephen, Ph.D. thesis, University of Massachusetts, 1970 (unpublished).

[36] Y. Kondo et al., Nucl. Phys. A676, 371 (2000).

[37] J. K. Ahn et al., Nucl. Phys. A648, 263 (1999).

[38] K. Tominaga et al., Nucl. Phys. A642, 483 (1998).

[39] A. Nogga et al., in preparation.

[40] K. Miyagawa and W. Glöckle, Phys. Rev. C 48, 2576 (1993). 
[41] K. Miyagawa, H. Kamada, W. Glöckle, and V. Stoks, Phys. Rev. C 51, 2905 (1995).

[42] H. Yamamura, K. Miyagawa, T. Mart, C. Bennhold, W. Glöckle, and H. Haberzettl, Phys. Rev. C 61, 014001 (1999).

[43] Y. Akaishi, T. Harada, S. Shinmura, and K. S. Myint, Phys. Rev. Lett. 84, 3539 (2000).

[44] A. Nogga, H. Kamada, and W. Glöckle, Phys. Rev. Lett. 88, 172501 (2002).

[45] H. Nemura, Y. Akaishi, and Y. Suzuki, Phys. Rev. Lett. 89, 142504 (2002).

[46] Y. Fujiwara, K. Miyagawa, M. Kohno, and Y. Suzuki, Phys. Rev. C 70, 024001 (2004).

[47] E. Hiyama, B. F. Gibson, and M. Kamimura, Phys. Rev. C 70, 031001(R) (2004).

[48] I. Vidana, A. Polls, A. Ramos, and H.-J. Schulze, Phys. Rev. C 64, 044301 (2001).

[49] Y. Tzeng, S. Y. Tsay Tzeng, and T. T. S. Kuo, Phys. Rev. C 65, 047303 (2002).
[50] S. Fujii, R. Okamoto, and K. Suzuki, Phys. Rev. C 66, 054301 (2002).

[51] C. M. Keil, H. Lenske, and C. Greiner, J. Phys. G 28, 1683 (2002).

[52] C. M. Vincent and S. C. Phatak, Phys. Rev. C 10, 391 (1974).

[53] Y. Fujiwara, M. Kohno, C. Nakamoto, and Y. Suzuki, Phys. Rev. C 64, 054001 (2001).

[54] E. M. Aitala et al., Phys. Rev. Lett. 89, 121801 (2002).

[55] K. Miyagawa, H. Kamada, W. Glöckle, H. Yamamura, T. Mart, and C. Bennhold, Few-Body Syst. Suppl. 12, 324 (2000).

[56] K. Miyagawa and H. Yamamura, Phys. Rev. C 60, 024003 (1999).

[57] Y. Fujiwara, C. Nakamoto, and Y. Suzuki, Phys. Rev. C 54, 2180 (1996).

[58] V. Bernard, T. R. Hemmert, and U.-G. Meißner, Phys. Lett. B565, 137 (2003).

[59] In a more modern language, it can be shown that such offshell parameters really correspond to low-energy constants of nonpropagating contact interactions, see, e.g., Ref. [58]. 